\title{
Application of new quintic polynomial B-spline approximation for numerical investigation of Kuramoto-Sivashinsky equation
}

\author{
Muhammad Kashif Iqbal ${ }^{1 *} \mathbb{D}$, Muhammad Abbas $^{2}$ (D) Tahir Nazir $^{2}$ and Nouman Ali ${ }^{3}$
}

\section{"Correspondence:}

kashifiqbal@gcuf.edu.pk

${ }^{1}$ Department of Mathematics,

Government College University,

Allama lqbal Road, 38000

Faisalabad, Pakistan

Full list of author information is

available at the end of the article

\section{Springer}

\begin{abstract}
A spline is a piecewise defined special function that is usually comprised of polynomials of a certain degree. These polynomials are supposed to generate a smooth curve by connecting at given data points. In this work, an application of fifth degree basis spline functions is presented for a numerical investigation of the Kuramoto-Sivashinsky equation. The finite forward difference formula is used for temporal integration, whereas the basis splines, together with a new approximation for fourth order spatial derivative, are brought into play for discretization in space direction. In order to corroborate the presented numerical algorithm, some test problems are considered and the computational results are compared with existing methods.
\end{abstract}

Keywords: Quintic polynomial B-spline functions; Crank-Nicolson scheme; Spline approximations; Von Neumann stability analysis; Kuramoto-Sivashinsky equation

\section{Introduction}

The Kuramoto-Sivashinsky (KS) equation, a canonical nonlinear evolution equation, crops up in mathematical modeling of several physical phenomena indicating reactiondiffusion systems, unstable drift waves in plasmas, pattern formation on thin hydrodynamic films, flame front instability, long waves on the interface between two viscous fluids, fluid flow on a vertical plate and spatially uniform oscillating chemical reaction in some homogeneous medium $[1,2]$. The KS equation has chaotic behavior and exhibits a traveling wave like solution that moves without changing its shape in a finite spatial domain [3-5]. The generalized KS equation is given by

$$
\begin{aligned}
& \frac{\partial y(x, t)}{\partial t}+y(x, t) \frac{\partial y(x, t)}{\partial x}+\alpha \frac{\partial^{2} y(x, t)}{\partial x^{2}}+\beta \frac{\partial^{3} y(x, t)}{\partial x^{3}}+\gamma \frac{\partial^{4} y(x, t)}{\partial x^{4}}=0, \\
& x \in[a, b], \quad t \in[0, T],
\end{aligned}
$$

(c) The Author(s) 2020. This article is licensed under a Creative Commons Attribution 4.0 International License, which permits use, sharing, adaptation, distribution and reproduction in any medium or format, as long as you give appropriate credit to the original author(s) and the source, provide a link to the Creative Commons licence, and indicate if changes were made. The images or other third party material in this article are included in the article's Creative Commons licence, unless indicated otherwise in a credit line to the material. If material is not included in the article's Creative Commons licence and your intended use is not permitted by statutory regulation or exceeds the permitted use, you will need to obtain permission directly from the copyright holder. To view a copy of this licence, visit http://creativecommons.org/licenses/by/4.0/. 
subject to the following conditions:

$$
\begin{aligned}
& y(x, 0)=\phi(x), \\
& \left\{\begin{array}{l}
y(a, t)=\phi_{1}(t), \quad y(b, t)=\phi_{2}(t), \\
y_{x}(a, t)=\psi_{1}(t), \quad y_{x}(b, t)=\psi_{2}(t),
\end{array}\right.
\end{aligned}
$$

where $y(x, t)$ gives the wave displacement at position $x$ and time $t, \alpha, \beta, \gamma$ are constants and $\phi(x), \phi_{i}(t), \psi_{i}(t)$ are known functions. The term $y_{x x}$ is responsible for instability at broad scales and the dissipative term $y_{x x x x}$ controls the damping effect at small scales. The nonlinear term $y y_{x}$ serves as an energy stabilizer by transmitting it between small and large scales [6]. The nonlinear evolution equations have attracted a considerable amount of research work in recent years [7-15]. Several numerical and analytical techniques have been proposed for solving these equations [16-23]. Khater and Temsah [24] employed the Chebyshev spectral collocation approach for an approximate solution of the generalized fourth order KS equation. Lai and Ma [25] proposed a lattice Boltzmann model for solving the nonlinear KS equation, A mesh free approach based on radial basis functions was used in [26] for an approximate solution of the generalized KS equation. Mittal and Arora [27] explored the numerical solution to KS equation by means of the Crank-Nicolson scheme and quintic B-spline (QnBS) functions. Porshokouhi and Ghanbari [28] implemented a variational iteration method for series solution of KS equation. The authors in [29] presented a numerical approach based on basis spline functions for an approximate solution of the KS equation. Rageh et al. [30] implemented a restrictive Taylor approximation method to find a numerical solution for the KS equation. Ersoy and Dag [31] proposed an exponential cubic B-spline method for numerical solution of KS equation. Mittal and Dahiya [6] proposed a differential quadrature method based on QnBS functions for solving the generalized KS equation. Gomes et al. [32] used linear feedback controls and techniques to stabilize the non-uniform unstable steady states of the generalized KS equation. The authors in [33] used polynomial scaling functions for solving the generalized KS equation. Akgul and Bonyah [34] proposed a reproducing kernel Hilbert space method for the solving generalized KS equation.

In this article, the numerical investigation of the nonlinear KS equation has been presented. The finite forward difference formulation and quintic polynomial basis spline functions are used to discretize the problem in time and spatial domains, respectively. The spatial order of convergence of a typical QnBS approximation scheme has been improved by involving a new approximation for the fourth order derivative. The stability of proposed algorithm has been studied by means of Von-Neumann stability analysis.

This study is organized as: In the first section, we discuss some basic ideas related to QnBS functions. The development of new approximation for $y^{4}(x)$ is explained in Sect. 3. The numerical method is discussed in Sect. 4. Section 5-6 consists of a stability and error analysis and finally the computational results are reported in Sect. 7.

\section{Quintic polynomial B-spline functions}

Let us partition the domain $[a, b]$ into $n$ intervals, $\left[x_{i}, x_{i+1}\right]$, of equal length such that $x_{i}=$ $a+(i \times h), i=0,1, \ldots, n, a=x_{0}, b=x_{n}$ and $h=\frac{1}{n}(b-a)$. The $r$ th polynomial B-spline of 
degree $q$, order $q+1$, is defined as [35]

$$
B_{q, r}(x)=L_{q, r} B_{q-1, r}(x)+\left(1-L_{q, r+1}\right) B_{q-1, r+1}(x), \quad x \in\left[x_{r}, x_{r+1+q}\right],
$$

where $q>0, L_{q, r}=\frac{\left(x-x_{r}\right)}{\left(x_{r}+q-x_{r}\right)}$ and

$$
B_{0, r}(x)= \begin{cases}1, & \text { if } x \in\left[x_{r}, x_{r+1}\right] \\ 0, & \text { otherwise }\end{cases}
$$

Using (5) -(4) with $q=5$, we get fifth degree basis spline functions [36]:

$$
\begin{aligned}
& B_{5, r}(x) \\
& =\frac{1}{120 h^{5}} \\
& \times \begin{cases}\left(x-x_{r-3}\right)^{5}, & x \in\left[x_{r-3}, x_{r-2}\right], \\
h^{5}+5 h^{4}\left(x-x_{r-2}\right)+10 h^{3}\left(x-x_{r-2}\right)^{2}+10 h^{2}\left(x-x_{r-2}\right)^{3} & \\
\quad+5 h\left(x-x_{r-2}\right)^{4}-5\left(x-x_{r-2}\right)^{5}, & x \in\left[x_{r-2}, x_{r-1}\right], \\
26 h^{5}+50 h^{4}\left(x-x_{r-1}\right)+20 h^{3}\left(x-x_{r-1}\right)^{2}-20 h^{2}\left(x-x_{r-1}\right)^{3} & \\
\quad-20 h\left(x-x_{r-1}\right)^{4}+10\left(x-x_{r-1}\right)^{5}, & x \in\left[x_{r-1}, x_{r}\right] \\
26 h^{5}+50 h^{4}\left(x_{r+1}-x\right)+20 h^{3}\left(x_{r+1}-x\right)^{2}-20 h^{2}\left(x_{r+1}-x\right)^{3} & \\
\quad-20 h\left(x_{r+1}-x\right)^{4}+10\left(x_{r+1}-x\right)^{5}, & x \in\left[x_{r}, x_{r+1}\right] \\
h^{5}+5 h^{4}\left(x_{r+2}-x\right)+10 h^{3}\left(x_{r+2}-x\right)^{2}+10 h^{2}\left(x_{r+2}-x\right)^{3} & \\
\quad+5 h\left(x_{r+2}-x\right)^{4}-5\left(x_{r+2}-x\right)^{5}, & x \in\left[x_{r+1}, x_{r+2}\right] \\
\left(x_{r+3}-x\right)^{5}, & x \in\left[x_{r+2}, x_{r+3}\right] \\
0 & \text { otherwise, }\end{cases}
\end{aligned}
$$

where $r=-2,-1,0, \ldots, n+2$. The QnBS approximation $Y(x)$ for a sufficiently smooth function $y(x)$ is given by

$$
Y(x)=\sum_{r=-2}^{n+2} \sigma_{r} B_{5, r}(x),
$$

where the $\sigma_{r}$ are to be calculated. Let $Y_{i}, m_{i}, M_{i}, T_{i}$ and $F_{i}$ represent the QnBS approximations for $y\left(x_{i}\right), y^{\prime}\left(x_{i}\right), y^{(2)}\left(x_{i}\right), y^{(3)}\left(x_{i}\right)$ and $y^{(4)}\left(x_{i}\right)$, respectively.

Using (6) and (7), we have

$$
\begin{aligned}
& Y_{i}=\frac{1}{120}\left(\sigma_{i-2}+26 \sigma_{i-1}+66 \sigma_{i}+26 \sigma_{i+1}+\sigma_{i+2}\right), \\
& m_{i}=\frac{1}{24 h}\left(-\sigma_{i-2}-10 \sigma_{i-1}+10 \sigma_{i+1}+\sigma_{i+2}\right), \\
& M_{i}=\frac{1}{6 h^{2}}\left(\sigma_{i-2}+2 \sigma_{i-1}-6 \sigma_{i}+2 \sigma_{i+1}+\sigma_{i+2}\right), \\
& T_{i}=\frac{1}{2 h^{3}}\left(-\sigma_{i-2}+2 \sigma_{i-1}-2 \sigma_{i+1}+\sigma_{i+2}\right),
\end{aligned}
$$




$$
F_{i}=\frac{1}{h^{4}}\left(\sigma_{i-2}-4 \sigma_{i-1}+6 \sigma_{i}-4 \sigma_{i+1}+\sigma_{i+2}\right)
$$

Moreover, from (8)-(12), we establish the following relations [37-39]:

$$
\begin{aligned}
& m_{i}=y^{\prime}\left(x_{i}\right)+\frac{h^{6}}{5040} y^{(7)}\left(x_{i}\right)-\frac{h^{8}}{21,600} y^{(9)}\left(x_{i}\right)+\cdots, \\
& M_{i}=y^{\prime \prime}\left(x_{i}\right)+\frac{h^{4}}{720} y^{(6)}\left(x_{i}\right)-\frac{h^{6}}{3360} y^{(8)}\left(x_{i}\right)+\cdots, \\
& T_{i}=y^{(3)}\left(x_{i}\right)-\frac{h^{4}}{240} y^{(7)}\left(x_{i}\right)+\frac{11 h^{6}}{30,240} y^{(9)}\left(x_{i}\right)+\cdots, \\
& F_{i}=y^{(4)}\left(x_{i}\right)-\frac{h^{2}}{12} y^{(6)}\left(x_{i}\right)+\frac{h^{4}}{240} y^{(8)}\left(x_{i}\right)+\cdots
\end{aligned}
$$

We see that the truncation error in $F_{i}$ is $O\left(h^{2}\right)$. Instead of using (12), the authors in [36, 40] proposed a new $O\left(h^{3}\right)$ accurate approximation for the fourth order derivative. For the sake of completeness, we reproduce those results in the following section.

\section{Derivation of new approximation for $y^{(4)}(x)$}

From (16), we establish the following relation for $F_{i-2}$ at the $\operatorname{knot} x_{i},(i=2,3,4, \ldots, n-2)$ :

$$
\begin{aligned}
F_{i-2} & =y^{(4)}\left(x_{i-2}\right)-\frac{h^{2}}{12} y^{(6)}\left(x_{i-2}\right)+\frac{h^{4}}{240} y^{(8)}\left(x_{i-2}\right)+\cdots \\
& =y^{(4)}\left(x_{i}\right)-2 h y^{(5)}\left(x_{i}\right)+\frac{23 h^{2}}{12} y^{(6)}\left(x_{i}\right)-\frac{7 h^{3}}{6} y^{(7)}\left(x_{i}\right)+\cdots
\end{aligned}
$$

Similar expressions for $F_{i-1}, F_{i+1}$ and $F_{i+2}$ at $x_{i}$ are derived as follows:

$$
\begin{aligned}
& F_{i-1}=y^{(4)}\left(x_{i}\right)-h y^{(5)}\left(x_{i}\right)+\frac{5 h^{2}}{12} y^{(6)}\left(x_{i}\right)-\frac{h^{3}}{12} y^{(7)}\left(x_{i}\right)+\cdots \\
& F_{i+1}=y^{(4)}\left(x_{i}\right)+h y^{(5)}\left(x_{i}\right)+\frac{5 h^{2}}{12} y^{(6)}\left(x_{i}\right)+\frac{h^{3}}{12} y^{(7)}\left(x_{i}\right)+\cdots \\
& F_{i+2}=y^{(4)}\left(x_{i}\right)+2 h y^{(5)}\left(x_{i}\right)+\frac{23 h^{2}}{12} y^{(6)}\left(x_{i}\right)+\frac{7 h^{3}}{6} y^{(7)}\left(x_{i}\right)+\cdots
\end{aligned}
$$

Suppose $\tilde{F}_{i}$ denotes the new approximation for $y^{(4)}\left(x_{i}\right)$ s.t.

$$
\widetilde{F}_{i}=a_{1} F_{i-2}+a_{2} F_{i-1}+a_{3} F_{i}+a_{4} F_{i+1}+a_{5} F_{i+2} .
$$

Using (17)-(20) in (21), we obtain $a_{1}=-\frac{1}{240}, a_{2}=\frac{1}{10}, a_{3}=\frac{97}{120}, a_{4}=\frac{1}{10}$ and $a_{5}=-\frac{1}{240}$.

Hence

$$
\begin{aligned}
\widetilde{F}_{i}= & \frac{1}{240 h^{4}}\left(-\sigma_{i-4}+28 \sigma_{i-3}+92 \sigma_{i-2}-604 \sigma_{i-1}+970 \sigma_{i}-604 \sigma_{i+1}\right. \\
& \left.+92 \sigma_{i+2}+28 \sigma_{i+3}-\sigma_{i+4}\right), \quad i=2,3, \ldots, n-2 .
\end{aligned}
$$

For $x=x_{0}$, we consider

$$
\widetilde{F}_{0}=a_{1} F_{0}+a_{2} F_{1}+a_{3} F_{2}+a_{4} F_{3}
$$


where

$$
\begin{aligned}
& F_{0}=y^{(4)}\left(x_{0}\right)-\frac{h^{2}}{12} y^{(6)}\left(x_{0}\right)+\frac{h^{4}}{240} y^{(8)}\left(x_{0}\right)+\cdots, \\
& F_{1}=y^{(4)}\left(x_{0}\right)+h y^{(5)}+\frac{5 h^{2}}{12} y^{(6)}\left(x_{0}\right)+\frac{h^{3}}{12} y^{(7)}\left(x_{0}\right)+\cdots, \\
& F_{2}=y^{(4)}\left(x_{0}\right)+2 h y^{(5)}+\frac{23 h^{2}}{12} y^{(6)}\left(x_{0}\right)+\frac{7 h^{3}}{6} y^{(7)}\left(x_{0}\right)+\cdots, \\
& F_{3}=y^{(4)}\left(x_{0}\right)+3 h y^{(5)}+\frac{53 h^{2}}{12} y^{(6)}\left(x_{0}\right)+\frac{17 h^{3}}{4} y^{(7)}\left(x_{0}\right)+\cdots
\end{aligned}
$$

From (23)-(27), we get $a_{1}=\frac{7}{6}, a_{2}=-\frac{5}{12}, a_{3}=\frac{1}{3}$ and $a_{4}=-\frac{1}{12}$ to obtain the following relation:

$$
\widetilde{F}_{0}=\frac{1}{12 h^{4}}\left(14 \sigma_{-2}-61 \sigma_{-1}+108 \sigma_{0}-103 \sigma_{1}+62 \sigma_{2}-27 \sigma_{3}+8 \sigma_{4}-\sigma_{5}\right) .
$$

Working in similar way, the following relations can be derived at $x_{1}, x_{n-1}$ and $x_{n}$ :

$$
\begin{aligned}
\widetilde{F}_{1}= & \frac{1}{12 h^{4}}\left(\sigma_{-2}+6 \sigma_{-1}-33 \sigma_{0}+52 \sigma_{1}-33 \sigma_{2}+6 \sigma_{3}+\sigma_{4}\right), \\
\widetilde{F}_{n-1} & =\frac{1}{12 h^{4}}\left(\sigma_{n-4}+6 \sigma_{n-3}-33 \sigma_{n-2}+52 \sigma_{n-1}-33 \sigma_{n}+6 \sigma_{n+1}+\sigma_{n+2}\right), \\
\widetilde{F}_{n}= & \frac{1}{12 h^{4}}\left(-\sigma_{n-5}+8 \sigma_{n-4}-27 \sigma_{n-3}+62 \sigma_{n-2}\right. \\
& \left.-103 \sigma_{n-1}+108 \sigma_{n}-61 \sigma_{n+1}+14 \sigma_{n+2}\right) .
\end{aligned}
$$

\section{Description of the numerical method}

Applying a finite forward difference formula and $\theta$ weighted scheme in the time direction, the semi-discretized form of problem (1) is obtained as follows:

$$
\begin{gathered}
\frac{y^{j+1}-y^{j}}{\Delta t}+\theta\left[\left(y y_{x}\right)^{j+1}+\alpha y_{x x}^{j+1}+\beta y_{x x x}^{j+1}+\gamma y_{x x x x}^{j+1}\right] \\
+(1-\theta)\left[y^{j} y_{x}^{j}+\alpha y_{x x}^{j}+\beta y_{x x x}^{j+1}+\gamma y_{x x x x}^{j}\right]=0,
\end{gathered}
$$

where $\Delta t$ is the mesh size in the time direction, $0 \leq \theta \leq 1$ and $y^{j+1}$ is used to denote $y\left(x, t_{j}+\right.$ $\Delta t)$. The nonlinear term $\left(y y_{x}\right)^{j+1}$ is treated as [33]

$$
\left(y y_{x}\right)^{j+1}=y^{j+1} y_{x}^{j}+y^{j} y_{x}^{j+1}-y^{j} y_{x}^{j} .
$$

Substituting (33) into (32), we get

$$
\begin{aligned}
& \frac{y^{j+1}-y^{j}}{\Delta t}+\theta\left[y^{j+1} y_{x}^{j}+y^{j} y_{x}^{j+1}-y^{j} y_{x}^{j}+\alpha y_{x x}^{j+1}+\beta y_{x x x}^{j+1}+\gamma y_{x x x x}^{j+1}\right] \\
& +(1-\theta)\left[y^{j} y_{x}^{j}+\alpha y_{x x}^{j}+\beta y_{x x x}^{j}+\gamma y_{x x x x}^{j}\right]=0 .
\end{aligned}
$$


For $\theta=\frac{1}{2}$, Eq. (34) can be rearranged as

$$
\begin{aligned}
& {\left[\frac{2}{\Delta t}+y_{x}^{j}\right] y^{j+1}+y^{j} y_{x}^{j+1}+\alpha y_{x x}^{j+1}+\beta y_{x x x}^{j+1}+\gamma y_{x x x x}^{j+1}} \\
& =\frac{2}{\Delta t} y^{j}-\alpha y_{x x}^{j}-\beta y_{x x x}^{j}-\gamma y_{x x x x}^{j} .
\end{aligned}
$$

Now, let us divide the spatial domain $[a, b]$ in $n$ equal parts $\left[x_{i}, x_{i+1}\right]$ s.t. $x_{i}=x_{0}+i \times h$, $i=0,1, \ldots, n, a=x_{0}, b=x_{n}$ and $h=\frac{1}{n}(b-a)$.

Let $Y\left(x, t_{j}\right)$ be the QnBS solution for $(1)$ at $t=t_{j}$ s.t.

$$
Y\left(x, t_{j}\right)=\sum_{r=-2}^{n+2} \sigma_{r}^{j} B_{5, r}(x)
$$

where the $\sigma_{r}^{j}$ are unknown control points. Substituting (36) into (35), at $x=x_{i}$, we obtain

$$
w_{i}^{j} Y_{i}^{j+1}+Y_{i}^{j} m_{i}^{j+1}+\alpha M_{i}^{j+1}+\beta T_{i}^{j+1}+\gamma F_{i}^{j+1}=z_{i}^{j}
$$

where $w_{i}^{j}=\frac{2}{\Delta t}+m_{i}^{j}$ and $z_{i}^{j}=\frac{2}{\Delta t} Y_{i}^{j}-\alpha M_{i}^{j}-\beta T_{i}^{j}-\gamma F_{i}^{j}$.

Using (8)-(11), (22), (28) and (29)-(31) in (37), for $i=0,1,2,3, \ldots, n$, we get $n+1$ linear equations involving $n+5$ control points:

$$
\begin{aligned}
& \frac{w_{0}^{j}}{120}\left(\sigma_{-2}^{j+1}+26 \sigma_{-1}^{j+1}+66 \sigma_{0}^{j+1}+26 \sigma_{1}^{j+1}+\sigma_{2}^{j+1}\right)-\frac{Y_{0}^{j}}{24 h}\left(\sigma_{-2}^{j+1}+10 \sigma_{-1}^{j+1}-10 \sigma_{1}^{j+1}-\sigma_{2}^{j+1}\right) \\
& +\frac{\alpha}{6 h^{2}}\left(\sigma_{-2}^{j+1}+2 \sigma_{-1}^{j+1}-6 \sigma_{0}^{j+1}+2 \sigma_{1}^{j+1}+\sigma_{2}^{j+1}\right)-\frac{\beta}{3 h^{3}}\left(\sigma_{-2}^{j+1}-2 \sigma_{-1}^{j+1}+2 \sigma_{1}^{j+1}-\sigma_{2}^{j+1}\right) \\
& +\frac{\gamma}{12 h^{4}}\left(14 \sigma_{-2}^{j+1}-61 \sigma_{-1}^{j+1}+108 \sigma_{0}^{j+1}-103 \sigma_{1}^{j+1}+62 \sigma_{2}^{j+1}-27 \sigma_{3}^{j+1}+8 \sigma_{4}^{j+1}-\sigma_{5}^{j+1}\right) \\
& =z_{0}^{j} \\
& \frac{w_{1}^{j}}{120}\left(\sigma_{-1}^{j+1}+26 \sigma_{0}^{j+1}+66 \sigma_{1}^{j+1}+26 \sigma_{2}^{j+1}+\sigma_{3}^{j+1}\right)-\frac{Y_{1}^{j}}{24 h}\left(\sigma_{-1}^{j+1}+10 \sigma_{0}^{j+1}-10 \sigma_{2}^{j+1}-\sigma_{3}^{j+1}\right) \\
& +\frac{\alpha}{6 h^{2}}\left(\sigma_{-1}^{j+1}+2 \sigma_{0}^{j+1}-6 \sigma_{1}^{j+1}+2 \sigma_{2}^{j+1}+\sigma_{3}^{j+1}\right)-\frac{\beta}{3 h^{3}}\left(\sigma_{-1}^{j+1}-2 \sigma_{0}^{j+1}+2 \sigma_{2}^{j+1}-\sigma_{3}^{j+1}\right) \\
& +\frac{\gamma}{12 h^{4}}\left(\sigma_{-2}^{j+1}+6 \sigma_{-1}^{j+1}-33 \sigma_{0}^{j+1}+52 \sigma_{1}^{j+1}-33 \sigma_{2}^{j+1}+6 \sigma_{3}^{j+1}+\sigma_{4}^{j+1}\right)=z_{1}^{j}, \\
& \frac{w_{i}^{j}}{120}\left(\sigma_{i-2}^{j+1}+26 \sigma_{i-1}^{j+1}+66 \sigma_{i}^{j+1}+26 \sigma_{i+1}^{j+1}+\sigma_{i+2}^{j+1}\right)-\frac{Y_{i}^{j}}{24 h}\left(\sigma_{i-2}^{j+1}+10 \sigma_{i-1}^{j+1}-10 \sigma_{i+1}^{j+1}-\sigma_{i+2}^{j+1}\right) \\
& +\frac{\alpha}{6 h^{2}}\left(\sigma_{i-2}^{j+1}+2 \sigma_{i-1}^{j+1}-6 \sigma_{i}^{j+1}+2 \sigma_{i+1}^{j+1}+\sigma_{i+2}^{j+1}\right)-\frac{\beta}{3 h^{3}}\left(\sigma_{i-2}^{j+1}-2 \sigma_{i-1}^{j+1}+2 \sigma_{i+1}^{j+1}-\sigma_{i+2}^{j+1}\right) \\
& -\frac{\gamma}{240 h^{4}}\left(\sigma_{i-4}^{j+1}-28 \sigma_{i-3}^{j+1}-92 \sigma_{i-2}^{j+1}+604 \sigma_{i-1}^{j+1}-970 \sigma_{i}^{j+1}\right. \\
& \left.+604 \sigma_{i+1}^{j+1}-92 \sigma_{i+2}^{j+1}-28 \sigma_{i+3}^{j+1}+\sigma_{i+4}^{j+1}\right) \\
& =z_{i}^{j}, \quad i=2,3,4, \ldots, n-2 \text {, }
\end{aligned}
$$




$$
\begin{aligned}
& \frac{w_{n-1}^{j}}{120}\left(\sigma_{n-3}^{j+1}+26 \sigma_{n-2}^{j+1}+66 \sigma_{n-1}^{j+1}+26 \sigma_{n}^{j+1}+\sigma_{n+1}^{j+1}\right)-\frac{Y_{n-1}^{j}}{24 h}\left(\sigma_{n-3}^{j+1}+10 \sigma_{n-2}^{j+1}-10 \sigma_{n}^{j+1}-\sigma_{n+1}^{j+1}\right) \\
& +\frac{\alpha}{6 h^{2}}\left(\sigma_{n-3}^{j+1}+2 \sigma_{n-2}^{j+1}-6 \sigma_{n-1}^{j+1}+2 \sigma_{n}^{j+1}+\sigma_{n+1}^{j+1}\right)-\frac{\beta}{3 h^{3}}\left(\sigma_{n-3}^{j+1}-2 \sigma_{n-2}^{j+1}+2 \sigma_{n}^{j+1}-\sigma_{n+1}^{j+1}\right) \\
& +\frac{\gamma}{12 h^{4}}\left(\sigma_{n-4}^{j+1}+6 \sigma_{n-3}^{j+1}-33 \sigma_{n-2}^{j+1}+52 \sigma_{n-1}^{j+1}-33 \sigma_{n}^{j+1}+6 \sigma_{n+1}^{j+1}+\sigma_{n+2}^{j+1}\right)=z_{n-1}^{j}, \\
& \frac{w_{n}^{j}}{120}\left(\sigma_{n-2}^{j+1}+26 \sigma_{n-1}^{j+1}+66 \sigma_{n}^{j+1}+26 \sigma_{n+1}^{j+1}+\sigma_{n+2}^{j+1}\right)-\frac{Y_{n}^{j}}{24 h}\left(\sigma_{n-2}^{j+1}+10 \sigma_{n-1}^{j+1}-10 \sigma_{n+1}^{j+1}-\sigma_{n+2}^{j+1}\right) \\
& \quad+\frac{\alpha}{6 h^{2}}\left(\sigma_{n-2}^{j+1}+2 \sigma_{n-1}^{j+1}-6 \sigma_{n}^{j+1}+2 \sigma_{n+1}^{j+1}+\sigma_{n+2}^{j+1}\right)-\frac{\beta}{3 h^{3}}\left(\sigma_{n-2}^{j+1}-2 \sigma_{n-1}^{j+1}+2 \sigma_{n+1}^{j+1}-\sigma_{n+2}^{j+1}\right) \\
& \quad-\frac{\gamma}{12 h^{4}}\left(\sigma_{n-5}-8 \sigma_{n-4}+27 \sigma_{n-3}-62 \sigma_{n-2}+103 \sigma_{n-1}-108 \sigma_{n}+61 \sigma_{n+1}+14 \sigma_{n+2}\right) \\
& =z_{n}^{j} .
\end{aligned}
$$

From the given end conditions (3), we get

$$
\begin{aligned}
& \left(\sigma_{-2}^{j+1}+26 \sigma_{-1}^{j+1}+66 \sigma_{0}^{j+1}+26 \sigma_{1}^{j+1}+\sigma_{2}^{j+1}\right) / 120=\phi_{1}\left(t_{j+1}\right), \\
& \left(-\sigma_{-2}^{j+1}-10 \sigma_{-1}^{j+1}+10 \sigma_{1}^{j+1}+\sigma_{2}^{j+1}\right) / 24 h=\psi_{1}\left(t_{j+1}\right), \\
& \left(-\sigma_{n-2}^{j+1}-10 \sigma_{n-1}^{j+1}+10 \sigma_{n+1}^{j+1}+\sigma_{n+2}^{j+1}\right) / 24 h=\psi_{2}\left(t_{j+1}\right), \\
& \left(\sigma_{n-2}^{j+1}+26 \sigma_{n-1}^{j+1}+66 \sigma_{n}^{j+1}+26 \sigma_{n+1}^{j+1}+\sigma_{n+2}^{j+1}\right) / 120=\phi_{2}\left(t_{j+1}\right) .
\end{aligned}
$$

The set of equations (38)-(46) can be written in matrix form as

$$
L \sigma^{j+1}=R
$$

where $L$ is the $(n+5) \times(n+5)$ coefficient matrix, $R$ is $(n+5) \times 1$ matrix and $\sigma^{j+1}=$ $\left[\begin{array}{lllll}\sigma_{-2}^{j+1} & \sigma_{-1}^{j+1} & \sigma_{0}^{j+1} & \cdots & \sigma_{n+2}^{j+1}\end{array}\right]^{T}$. Solving (47), we get $\sigma^{j+1}$ and put these control points into (36) to get the approximate solution at $(j+1)$ th time level. However, first we need to find $\sigma^{0}$, using the given initial condition, as follows (2):

$$
\begin{aligned}
& \left(-\sigma_{-2}^{0}-10 \sigma_{-1}^{0}+10 \sigma_{1}^{0}+\sigma_{2}^{0}\right) / 24 h=\phi^{\prime}\left(x_{0}\right), \\
& \left(\sigma_{-2}^{0}+2 \sigma_{-1}^{0}-6 \sigma_{0}^{0}+2 \sigma_{1}^{0}+\sigma_{2}^{0}\right) / 6 h^{2}=\phi^{\prime \prime}\left(x_{0}\right), \\
& \left(\sigma_{i-2}^{0}+26 \sigma_{i-1}^{0}+66 \sigma_{i}^{0}+26 \sigma_{i+1}^{0}+\sigma_{i+2}^{0}\right) / 120=\phi\left(x_{i}\right), \quad i=0,1, \ldots, n, \\
& \left(\sigma_{n-2}^{0}+2 \sigma_{n-1}^{0}-6 \sigma_{n}^{0}+2 \sigma_{n+1}^{0}+\sigma_{n+2}^{0}\right) / 6 h^{2}=\phi^{\prime \prime}\left(x_{n}\right), \\
& \left(-\sigma_{n-2}^{0}-10 \sigma_{n-1}^{0}+10 \sigma_{n+1}^{0}+\sigma_{n+2}^{0}\right) / 24 h=\phi^{\prime}\left(x_{n}\right) .
\end{aligned}
$$

In matrix form, we have

$$
L \sigma^{0}=R
$$

The matrix system (48) can easily be solved using for $\sigma^{0}$ using a modified form of Thomas algorithm. The numerical simulation is run in Mathematica 10. 


\section{Stability analysis}

Setting $y=\eta$ in the nonlinear term $y y_{x}$, Eq. (32) takes the following form:

$$
\begin{gathered}
\frac{y^{j+1}-y^{j}}{\Delta t}+\theta\left[\eta\left(y_{x}\right)^{j+1}+\alpha y_{x x}^{j+1}+\beta y_{x x x}^{j+1}+\gamma y_{x x x x}^{j+1}\right] \\
+(1-\theta)\left[\eta y_{x}^{j}+\alpha y_{x x}^{j}+\beta y_{x x x}^{j+1}+\gamma y_{x x x x}^{j}\right]=0 .
\end{gathered}
$$

Setting $\theta=0.5$, the fully discretized form of (49) is as follows:

$$
\begin{gathered}
Y_{i}^{j+1}+\frac{\Delta t}{2}\left[\eta m_{i}^{j+1}+\alpha M_{i}^{j+1}+\beta T_{i}^{j+1}+\gamma F_{i}^{j+1}\right] \\
=Y_{i}^{j}-\frac{\Delta t}{2}\left[\eta m_{i}^{j}+\alpha M_{i}^{j}+\beta T_{i}^{j+1}+\gamma F_{i}^{j}\right] .
\end{gathered}
$$

Using (8)-(11) and (22) in (50), we have

$$
\begin{aligned}
-d_{3} \sigma_{i-4}^{j+1} & +28 d_{3} \sigma_{i-3}^{j+1}+2\left(46 d_{3}-60 h d_{2}+20 h^{2} d_{1}-5 h^{3} d_{4}+2 h^{4}\right) \sigma_{i-2}^{j+1} \\
& +4\left(-151 d_{3}+60 h d_{2}+20 h^{2} d_{1}-25 h^{3} d_{4}+26 h^{4}\right) \sigma_{i-1}^{j+1} \\
& +2\left(485 d_{3}-120 h d_{1}+132 h^{4}\right) \sigma_{i}^{j+1} \\
& +4\left(-151 d_{3}-60 h d_{2}-20 h^{2} d_{1}+25 h^{3} d_{4}+26 h^{4}\right) \sigma_{i+1}^{j+1} \\
& +2\left(46 d_{3}+60 h d_{2}+20 h^{2} d_{1}+5 h^{3} d_{4}+2 h^{4}\right) \sigma_{i+2}^{j+1}+28 d_{3} \sigma_{i+3}^{j+1}-d_{3} \sigma_{i+4}^{j+1} \\
= & d_{3} \sigma_{i-4}^{j}-28 d_{3} \sigma_{i-3}^{j}+2\left(-46 d_{3}+60 h d_{2}-20 h^{2} d_{1}+5 h^{3} d_{4}+2 h^{4}\right) \sigma_{i-2}^{j} \\
& +4\left(151 d_{3}-60 h d_{2}-20 h^{2} d_{1}+25 h^{3} d_{4}+26 h^{4}\right) \sigma_{i-1}^{j} \\
& +2\left(-485 d_{3}+120 h d_{1}+132 h^{4}\right) \sigma_{i}^{j} \\
& +4\left(151 d_{3}+60 h d_{2}-20 h^{2} d_{1}-25 h^{3} d_{4}+26 h^{4}\right) \sigma_{i+1}^{j} \\
& +2\left(-46 d_{3}-60 h d_{2}-20 h^{2} d_{1}-5 h^{3} d_{4}+2 h^{4}\right) \sigma_{i+2}^{j}-28 d_{3} \sigma_{i+3}^{j}+d_{3} \sigma_{i+4}^{j},
\end{aligned}
$$

where $d_{1}=\alpha \Delta t, d_{2}=\beta \Delta t, d_{3}=\gamma \Delta t, d_{4}=\eta \Delta t$.

Now, following [27, 41], we substitute $\sigma_{i}^{j}=\xi^{j} \mathrm{e}^{i m \varphi}$ into (51):

$$
\begin{aligned}
& \xi^{j+1}\left[-d_{3} \mathrm{e}^{\iota(m-4) \varphi}+28 d_{3} \mathrm{e}^{\ell(m-3) \varphi}+2\left(46 d_{3}-60 h d_{2}+20 h^{2} d_{1}-5 h^{3} d_{4}+2 h^{4}\right) \mathrm{e}^{\imath(m-2) \varphi}\right. \\
& +4\left(-151 d_{3}+60 h d_{2}+20 h^{2} d_{1}-25 h^{3} d_{4}+26 h^{4}\right) \mathrm{e}^{\iota(m-1) \varphi} \\
& +2\left(485 d_{3}-120 h d_{1}+132 h^{4}\right) \mathrm{e}^{\imath m \varphi} \\
& +4\left(-151 d_{3}-60 h d_{2}-20 h^{2} d_{1}+25 h^{3} d_{4}+26 h^{4}\right) \mathrm{e}^{\imath(m+1) \varphi} \\
& +2\left(46 d_{3}+60 h d_{2}+20 h^{2} d_{1}+5 h^{3} d_{4}+2 h^{4}\right) \mathrm{e}^{\iota(m+2) \varphi} \\
& \left.+28 d_{3} \mathrm{e}^{\ell(m+3) \varphi}-d_{3} \mathrm{e}^{\iota(m+4) \varphi}\right] \\
& =\xi^{j}\left[d_{3} \mathrm{e}^{\iota(m-4) \varphi}-28 d_{3} \mathrm{e}^{\iota(m-3) \varphi}\right. \\
& +2\left(-46 d_{3}+60 h d_{2}-20 h^{2} d_{1}+5 h^{3} d_{4}+2 h^{4}\right) \mathrm{e}^{l(m-2) \varphi} \\
& +4\left(151 d_{3}-60 h d_{2}-20 h^{2} d_{1}+25 h^{3} d_{4}+26 h^{4}\right) \mathrm{e}^{\iota(m-1) \varphi} \\
& +2\left(-485 d_{3}+120 h d_{1}+132 h^{4}\right) \mathrm{e}^{\imath m \varphi}
\end{aligned}
$$




$$
\begin{aligned}
& +4\left(151 d_{3}+60 h d_{2}-20 h^{2} d_{1}-25 h^{3} d_{4}+26 h^{4}\right) \mathrm{e}^{\iota(m+1) \varphi} \\
& +2\left(-46 d_{3}-60 h d_{2}-20 h^{2} d_{1}-5 h^{3} d_{4}+2 h^{4}\right) \mathrm{e}^{\iota(m+2) \varphi} \\
& \left.-28 d_{3} \mathrm{e}^{\iota(m+3) \varphi}+d_{3} \mathrm{e}^{\iota(m+4) \varphi}\right]
\end{aligned}
$$

where $\iota=\sqrt{-1}, \varphi=\zeta h$ and $\zeta$ is the mode number.

After some simplification, (52) takes the following form:

$$
\begin{aligned}
\xi[- & d_{3} \cos 4 \varphi+28 d_{3} \cos 3 \varphi+2\left(46 d_{3}+20 h^{2} d_{1}+2 h^{4}\right) \cos 2 \varphi \\
& +2 \iota\left(60 h d_{2}+5 h^{3} d_{4}\right) \sin 2 \varphi+4\left(-151 d_{3}+20 h^{2} d_{1}+26 h^{4}\right) \cos \varphi \\
& \left.+4 \iota\left(-60 h d_{2}+25 h^{3} d_{4}\right) \sin \varphi+485 d_{3}-120 h d_{1}+132 h^{4}\right] \\
= & {\left[d_{3} \cos 4 \varphi-28 d_{3} \cos 3 \varphi+2\left(-46 d_{3}-20 h^{2} d_{1}+2 h^{4}\right) \cos 2 \varphi\right.} \\
& +2 \iota\left(-60 h d_{2}-5 h^{3} d_{4}\right) \sin 2 \varphi+4\left(151 d_{3}-20 h^{2} d_{1}+26 h^{4}\right) \cos \varphi \\
& \left.+4 \iota\left(60 h d_{2}-25 h^{3} d_{4}\right) \sin \varphi-485 d_{3}+120 h d_{1}+132 h^{4}\right]
\end{aligned}
$$

Equation (53) can be written as $\xi=\frac{\nu_{1}-i \omega}{v_{2}+i \omega}$, where

$$
\begin{aligned}
v_{1}= & d_{3} \cos 4 \varphi-28 d_{3} \cos 3 \varphi+2\left(-46 d_{3}-20 h^{2} d_{1}+2 h^{4}\right) \cos 2 \varphi \\
& +4\left(151 d_{3}-20 h^{2} d_{1}+26 h^{4}\right) \cos \varphi-485 d_{3}+120 h d_{1}+132 h^{4} \\
v_{2}= & -d_{3} \cos 4 \varphi+28 d_{3} \cos 3 \varphi+2\left(46 d_{3}+20 h^{2} d_{1}+2 h^{4}\right) \cos 2 \varphi \\
& +4\left(-151 d_{3}+20 h^{2} d_{1}+26 h^{4}\right) \cos \varphi+485 d_{3}-120 h d_{1}+132 h^{4} \\
\omega= & 2\left(-60 h d_{2}-5 h^{3} d_{4}\right) \sin 2 \varphi+4\left(60 h d_{2}-25 h^{3} d_{4}\right) \sin \varphi
\end{aligned}
$$

Now

$$
\begin{aligned}
v_{2}^{2}-v_{1}^{2}= & 64 h^{4}(33+26 \cos \varphi+\cos 2 \varphi) \sin ^{2} \frac{\varphi}{2}\left[170 d_{3}-80 d_{1} h^{2}\right. \\
& \left.-5\left(29 d_{3}+8 d_{1} h^{2}\right) \cos \varphi-26 d_{3} \cos 2 \varphi+d_{3} \cos 3 \varphi\right] .
\end{aligned}
$$

We plug in the values of $d_{1}$ and $d_{3}$ with $\alpha=-1, \gamma=1$ in the last expression to get

$$
\begin{aligned}
v_{2}^{2}-v_{1}^{2}= & 64 h^{4}(33+26 \cos \varphi+\cos 2 \varphi) \sin ^{2} \frac{\varphi}{2} \Delta t\left[(97+24 \cos \varphi-\cos 2 \varphi) \sin ^{2} \frac{\varphi}{2}\right. \\
& \left.+40 h^{2}(2+\cos \varphi)\right] .
\end{aligned}
$$

Since

$$
\begin{aligned}
& 64 h^{4}(33+26 \cos \varphi+\cos 2 \varphi) \sin ^{2} \frac{\varphi}{2} \Delta t \geq 0, \\
& (97+24 \cos \varphi-\cos 2 \varphi) \sin ^{2} \frac{\varphi}{2} \geq 0, \\
& \left.40 h^{2}(2+\cos \varphi)\right] \geq 0,
\end{aligned}
$$

we have $v_{2}^{2}-v_{1}^{2} \geq 0$. 
Consequently $|\xi| \leq 1$, the proposed algorithm is proved to be stable.

\section{Error analysis}

From (8)-(11), we can obtain the following expressions [38, 39]:

$$
\begin{aligned}
& h\left[Y^{\prime}\left(x_{i-2}\right)+26 Y^{\prime}\left(x_{i-1}\right)+66 Y^{\prime}\left(x_{i}\right)+26 Y^{\prime}\left(x_{i+1}\right)+Y^{\prime}\left(x_{i+2}\right)\right] \\
& \quad=5\left[-Y\left(x_{i-2}\right)-10 Y\left(x_{i-1}\right)+10 Y\left(x_{i+1}\right)+Y\left(x_{i+2}\right)\right], \\
& h^{2}\left[Y^{\prime \prime}\left(x_{i-2}\right)+26 Y^{\prime \prime}\left(x_{i-1}\right)+66 Y^{\prime \prime}\left(x_{i}\right)+26 Y^{\prime \prime}\left(x_{i+1}\right)+Y^{\prime \prime}\left(x_{i+2}\right)\right] \\
& \quad=20\left[Y\left(x_{i-2}\right)+2 Y\left(x_{i-1}\right)-6 Y\left(x_{i}\right)+2 Y\left(x_{i+1}\right)+Y\left(x_{i+2}\right)\right], \\
& h^{3}\left[Y^{\prime \prime \prime}\left(x_{i-2}\right)+26 Y^{\prime \prime \prime}\left(x_{i-1}\right)+66 Y^{\prime \prime \prime}\left(x_{i}\right)+26 Y^{\prime \prime \prime}\left(x_{i+1}\right)+Y^{\prime \prime \prime}\left(x_{i+2}\right)\right] \\
& \quad=60\left[-Y\left(x_{i-2}\right)+2 Y\left(x_{i-1}\right)-2 Y\left(x_{i+1}\right)+Y\left(x_{i+2}\right)\right] .
\end{aligned}
$$

Similarly, using (10), (11) and (22), we have

$$
\begin{aligned}
h^{4} Y^{(4)}\left(x_{i}\right)= & \frac{h^{2}}{40}\left[-Y^{\prime \prime}\left(x_{i-2}\right)+114 Y^{\prime \prime}\left(x_{i-1}\right)-142 Y^{\prime \prime}\left(x_{i}\right)+30 Y^{\prime \prime}\left(x_{i+1}\right)-Y^{\prime \prime}\left(x_{i+2}\right)\right] \\
& +\frac{7 h^{3}}{10}\left[Y^{\prime \prime \prime}\left(x_{i-1}\right)+2 Y^{\prime \prime \prime}\left(x_{i}\right)\right] .
\end{aligned}
$$

Employing the operator notation, $E^{\lambda}\left(Y^{\prime}\left(x_{i}\right)\right)=Y^{\prime}\left(x_{i+\lambda}\right), \lambda \in \mathbb{Z}$, Eqs. (54)-(56) are written as [37]

$$
\begin{aligned}
& h\left[E^{-2}+26 E^{-1}+66+26 E^{1}+E^{2}\right] Y^{\prime}\left(x_{i}\right)=5\left[-E^{-2}-10 E^{-1}+10 E^{1}+E^{2}\right] y\left(x_{i}\right), \\
& h^{2}\left[E^{-2}+26 E^{-1}+66+26 E^{1}+E^{2}\right] Y^{\prime \prime}\left(x_{i}\right)=20\left[E^{-2}-2 E^{-1}+-6+2 E^{1}+E^{2}\right] y\left(x_{i}\right), \\
& h^{3}\left[E^{-2}+26 E^{-1}+66+26 E^{1}+E^{2}\right] Y^{\prime \prime \prime}\left(x_{i}\right)=60\left[-E^{-2}+2 E^{-1}-2 E^{1}+E^{2}\right] y\left(x_{i}\right) .
\end{aligned}
$$

Using $E=\mathrm{e}^{h D}, D \equiv d / d x$, Eqs. (58)-(60) give the following expressions, respectively [38, 39]:

$$
\begin{aligned}
& Y^{\prime}\left(x_{i}\right)=y^{\prime}\left(x_{i}\right)+\frac{h^{6}}{5040} y^{(7)}\left(x_{i}\right)-\frac{h^{8}}{21,600} y^{(9)}\left(x_{i}\right)+\frac{h^{10}}{1,036,800} y^{(11)}\left(x_{i}\right)+\cdots, \\
& Y^{\prime \prime}\left(x_{i}\right)=y^{\prime \prime}\left(x_{i}\right)+\frac{h^{4}}{720} y^{(6)}\left(x_{i}\right)-\frac{h^{6}}{3360} y^{(8)}\left(x_{i}\right)+\frac{h^{8}}{86,400} y^{(10)}\left(x_{i}\right)+\cdots, \\
& Y^{\prime \prime \prime}\left(x_{i}\right)=y^{(3)}\left(x_{i}\right)-\frac{h^{4}}{240} y^{(7)}\left(x_{i}\right)+\frac{11 h^{6}}{30,240} y^{(9)}\left(x_{i}\right)-\frac{h^{8}}{288,00} y^{(11)}\left(x_{i}\right)+\cdots
\end{aligned}
$$

Similarly, writing (57) in operator notation, we get

$$
h^{4} Y^{(4)}\left(x_{i}\right)=\frac{h^{2}}{40}\left[-E^{-2}+114 E^{-1}-142+30 E^{1}-E^{2}\right] y^{\prime \prime}\left(x_{i}\right)+\frac{7 h^{3}}{10}\left[E^{-1}+2\right] y^{(3)}\left(x_{i}\right)
$$


The above relation can be expanded as

$$
\begin{aligned}
h^{4} Y^{(4)}\left(x_{i}\right)= & \frac{h^{2}}{40}\left[-84 h D+68 h^{2} D^{2}-14 h^{3} D^{3}+\frac{14}{3} h^{4} D^{4}+\cdots\right] y^{\prime \prime}\left(x_{i}\right) \\
& +\frac{7 h^{3}}{10}\left[3-h D+\frac{1}{2} h^{2} D^{2}-\frac{1}{6} h^{3} D^{3}+\frac{1}{24} h^{4} D^{4}\right] y^{(3)}\left(x_{i}\right) .
\end{aligned}
$$

After some simplification, we obtain

$$
Y^{(4)}\left(x_{i}\right)=y^{(4)}\left(x_{i}\right)+\frac{7 h^{3}}{600} y^{(7)}\left(x_{i}\right)-\frac{19 h^{4}}{3600} y^{(8)}\left(x_{i}\right)+\cdots .
$$

Now, the generalized KS equation (1) can be written as

$$
y_{t}=G(x, t, y)
$$

where $G=-y y_{x}-\alpha y_{x x}-\beta y_{x x x}-\gamma y_{x x x x}$, with $y_{i}^{j+1}-y_{i}^{j}=\Delta t\left[\theta G_{i}^{j+1}+(1-\theta) G_{i}^{j}\right]$.

Applying a Taylor series about $(j+\theta) \Delta t$, we obtain

$$
\begin{aligned}
\left(y_{t}\right)_{i} & -\frac{2 \theta-1}{2} \Delta t\left(y_{t t}\right)_{i}+\frac{1+3 \theta(\theta-1)}{6} \Delta t^{2}\left(y_{t t t}\right)_{i}+\cdots \\
= & G_{i}-\frac{\theta(\theta-1)}{2} \Delta t^{2}\left(G_{t t}\right)_{i}+\frac{\theta(\theta-1)(2 \theta-1)}{2} \Delta t^{3}\left(G_{t t t}\right)_{i}+\cdots .
\end{aligned}
$$

Setting $y=\eta$ in the nonlinear term $y y_{x}$ and using (67) in (68), we get

$$
\left(y_{t}\right)_{i}=G_{i}+\frac{2 \theta-1}{2} \Delta t\left(G_{t}\right)_{i}-\frac{1+6 \theta(\theta-1)}{6} \Delta t^{2}\left(G_{t t}\right)_{i}+\cdots .
$$

From (67), the truncation error is defined as

$$
\begin{aligned}
& e_{i}=\left(y_{t}\right)_{i}-\left[-\eta\left(Y_{x}\right)_{i}-\alpha\left(Y_{x x}\right)_{i}-\beta\left(Y_{x x x}\right)_{i}-\gamma\left(Y_{x x x x}\right)_{i}\right], \\
& e_{i}=\frac{2 \theta-1}{2} \Delta t\left(y_{t t}\right)_{i}+\frac{7 \gamma h^{3}}{600}\left(y_{x x x x x x x}\right)_{i}+\cdots .
\end{aligned}
$$

Hence, theoretically, the proposed numerical algorithm for KS equation is $O\left(\Delta t+h^{3}\right)$ convergent.

\section{Numerical results}

To show the versatility of numerical algorithm, we have presented four numerical experiments. The accuracy and efficiency of the method is tested by the maximum, Euclidian and the global relative error (GRE) norms, which are calculated as [27, 42]

$$
L_{\infty}=\max _{0 \leq i \leq n}\left|y_{i}-Y_{i}\right|, \quad L_{2}=\sqrt{h \sum_{i=0}^{n}\left(y_{i}-Y_{i}\right)^{2}}, \quad \text { GRE }=\frac{\sum_{i=0}^{n}\left|y_{i}-Y_{i}\right|}{\sum_{i=0}^{n}\left|y_{i}\right|},
$$

where $Y_{i}$ and $y_{i}$ are the approximate and exact solutions at the $i$ th spatial knot, respectively. The numerical outcomes are compared with the Lattice Boltzmann model (LBM) [25], the Quintic B-spline collocation method (QnBSM) [27], B-spline functions (BSF) [29], 
the Exponential cubic B-spline collocation method (ExCBSM) [31], the QnBS differential quadrature method (QnBS-DQM) [6] and Polynomial scaling functions (PSF) [33].

Problem 1 Consider the following KS equation [6, 25, 27, 31]:

$$
y_{t}+y y_{x}+y_{x x}+y_{x x x x}=0, \quad x \in[-30,30], t \in[0,4] .
$$

The piecewise defined spline solution at $t=1$ using the proposed method for Example 1, when $n=100, \Delta t=0.01, \lambda=5$ and $v=-12$, is given by

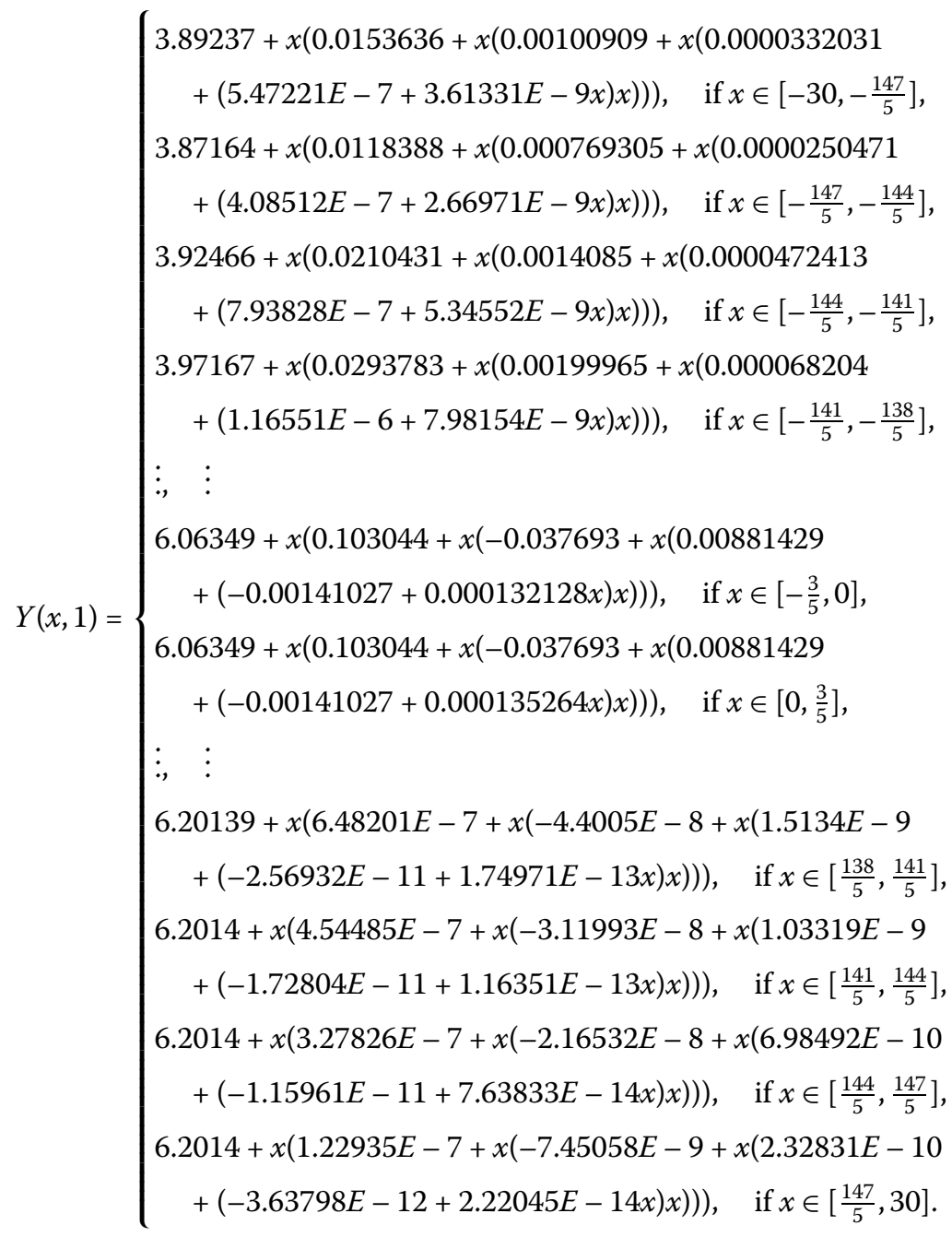

The exact solution is

$$
y(x, t)=\lambda+\frac{30}{19} \mu\left[-9 \tanh \left(\mu(x-\lambda t-v)+11 \tanh ^{3}(\mu(x-\lambda t-v))\right],\right.
$$

where $\mu=\frac{1}{2} \sqrt{\frac{11}{19}}$ and the initial and end conditions can be derived from the given exact solution. The GRE corresponding to $\lambda=5, v=-12, n=100$ and $\Delta t=0.01$ is listed in Table 1 at $t=1,2,3,4$. It can be observed that our approximate results are better than LBM [25], QnBSM [27], ExCBSM [31] and QnBS-DQM [6]. Table 2 shows a comparison of computational error norms with QnBS-DQM [6] corresponding to $\lambda=0.1, v=-10$, 
Table 1 Global relative error for Problem 1 when $\lambda=5$ and $v=-12$

\begin{tabular}{llllll}
\hline$t$ & LBM [25] & QnBSM [27] & ExCBSM [31] & QnBS-DQM [6] & $\begin{array}{l}\text { Proposed method } \\
n=100\end{array}$ \\
& $n=600$ & $n=150$ & $n=150$ & $n=150$ & $\Delta t=0.01$ \\
\hline 1 & $\Delta t=0.0001$ & $\Delta t=0.01$ & $\Delta t=0.01$ & & $2.50 \times 10^{-5}$ \\
2 & $6.79 \times 10^{-4}$ & $3.82 \times 10^{-4}$ & $3.33 \times 10^{-4}$ & $2.40 \times 10^{-4}$ & $4.57 \times 10^{-5}$ \\
3 & $1.15 \times 10^{-3}$ & $5.51 \times 10^{-4}$ & $5.56 \times 10^{-4}$ & $2.99 \times 10^{-4}$ & $6.35 \times 10^{-5}$ \\
4 & $1.59 \times 10^{-3}$ & $7.04 \times 10^{-4}$ & $8.75 \times 10^{-4}$ & $3.63 \times 10^{-4}$ & $7.86 \times 10^{-5}$ \\
\hline
\end{tabular}

Table $2 L_{2}$ and $L_{\infty}$ norms for Problem 1 when $\lambda=0.1$ and $v=-10$

\begin{tabular}{llllll}
\hline$t$ & QnBS-DQM [6] & & & \multicolumn{2}{l}{ Proposed method } \\
\cline { 2 - 3 } \cline { 6 - 6 } & $L_{2}$ & & & $L_{\infty}$ & $L_{\infty}$ \\
\hline 0.1 & $8.50 \times 10^{-3}$ & $8.59 \times 10^{-3}$ & & $1.74 \times 10^{-4}$ & $1.17 \times 10^{-4}$ \\
0.3 & $1.18 \times 10^{-2}$ & $9.52 \times 10^{-3}$ & & $3.36 \times 10^{-4}$ & $2.12 \times 10^{-4}$ \\
0.5 & $1.44 \times 10^{-2}$ & $1.00 \times 10^{-2}$ & & $4.72 \times 10^{-4}$ & $2.83 \times 10^{-4}$ \\
0.7 & $1.69 \times 10^{-2}$ & $1.04 \times 10^{-2}$ & & $5.83 \times 10^{-4}$ & $3.35 \times 10^{-4}$ \\
1.0 & $1.92 \times 10^{-2}$ & $1.19 \times 10^{-2}$ & & $7.14 \times 10^{-4}$ & $3.87 \times 10^{-4}$ \\
\hline
\end{tabular}

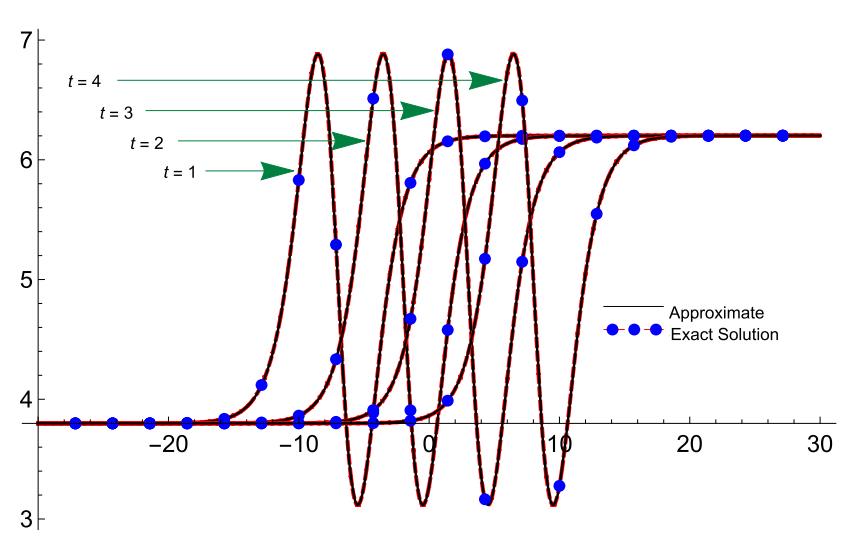

Figure 1 Numerical and exact solution for Problem 1 using $n=100, \Delta t=0.01, \lambda=5$ and $v=-12$

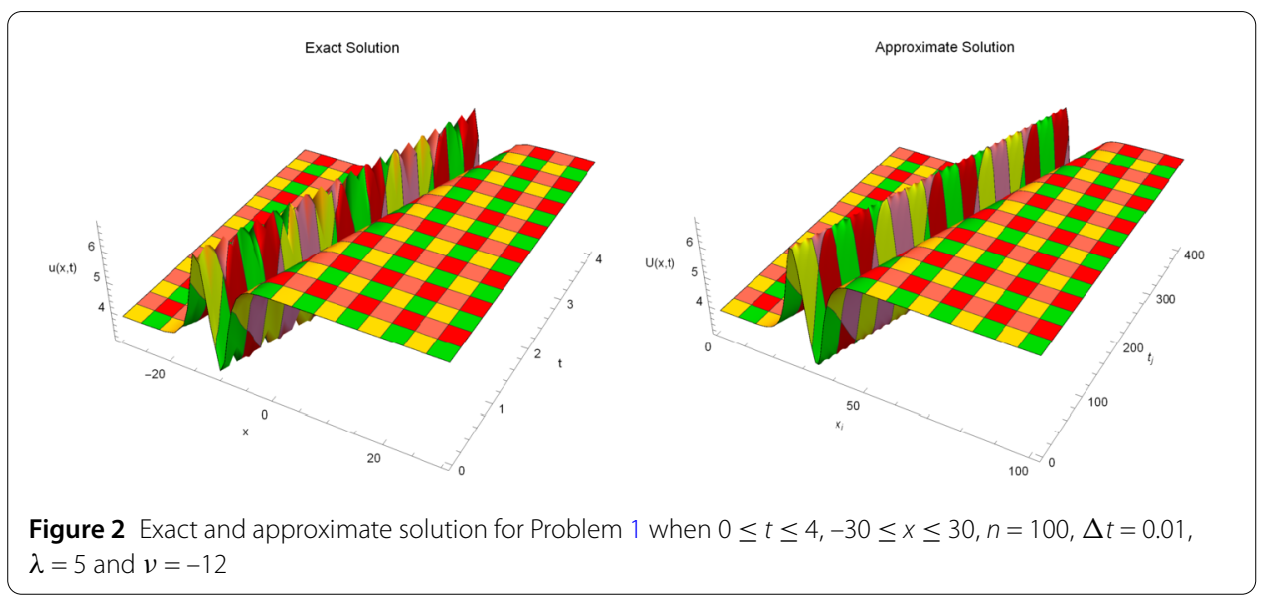

$n=100$ and $\Delta t=0.01$ at $t=0.1,0.3,0.5,0.5,0.7,1.0$. The 2D plots of approximate and exact solutions at different time stages are displayed in Fig. 1 and the 3D graphics of the exact and numerical solutions are portrayed in Fig. 2. 
Problem 2 Consider the following KS equation [6, 25, 27, 31]:

$$
y_{t}+y y_{x}-y_{x x}+y_{x x x x}=0, \quad x \in[-50,50], t \in[0,4] .
$$

The piecewise defined spline solution at $t=1$ using the proposed method for Example 2, when $n=100, \Delta t=0.01, \lambda=5$ and $v=-25$, is given by

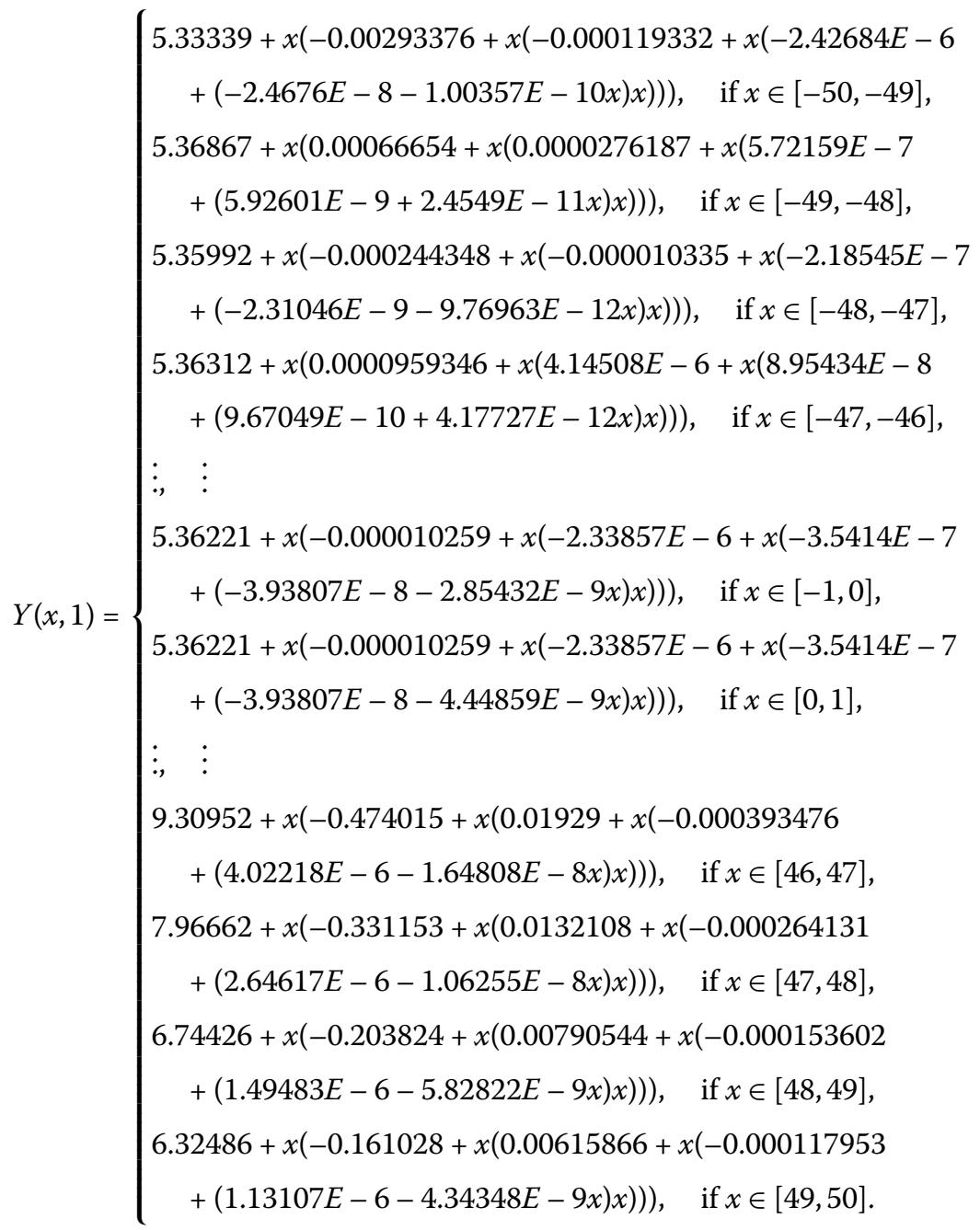

The exact solution is

$$
y(x, t)=\lambda+\frac{30}{19} \mu\left[-3 \tanh (\mu(x-\lambda t-v))+\tanh ^{3}(\mu(x-\lambda t-v))\right],
$$

where $\mu=\frac{1}{2 \sqrt{19}}$ and the initial and boundary constraints can be derived from given exact solution. In Table 3, the GRE corresponding to $\lambda=5, v=-25, n=100$ and $\Delta t=$ 0.01 is compared with LBM [25], QnBSM [27], ExCBSM [31] and QnBS-DQM [6] at $t=6,8,10,12$. Figure 3 exhibits $2 \mathrm{D}$ plots of the exact and numerical solution at dif- 
Table 3 Global relative error for Problem 2 when $\lambda=5$ and $\nu=-25$

\begin{tabular}{rlllll}
\hline$t$ & LBM [25] & QnBSM [27] & ExCBSM [31] & QnBS-DQM [6] & $\begin{array}{l}\text { Proposed method } \\
n=100\end{array}$ \\
& $n=1000$ & $n=200$ & $n=150$ & $\Delta t=0.01$ \\
\hline 6 & $\Delta t=0.0001$ & $\Delta t=0.01$ & $\Delta t=0.01$ & & $3.00 \times 10^{-7}$ \\
8 & $7.88 \times 10^{-6}$ & $6.51 \times 10^{-6}$ & $9.34 \times 10^{-6}$ & $3.59 \times 10^{-6}$ & $3.62 \times 10^{-7}$ \\
10 & $9.53 \times 10^{-6}$ & $7.13 \times 10^{-6}$ & $1.57 \times 10^{-5}$ & $4.29 \times 10^{-6}$ & $4.14 \times 10^{-7}$ \\
12 & $1.09 \times 10^{-5}$ & $7.31 \times 10^{-6}$ & $2.37 \times 10^{-5}$ & $5.09 \times 10^{-6}$ & $4.43 \times 10^{-7}$ \\
\hline
\end{tabular}

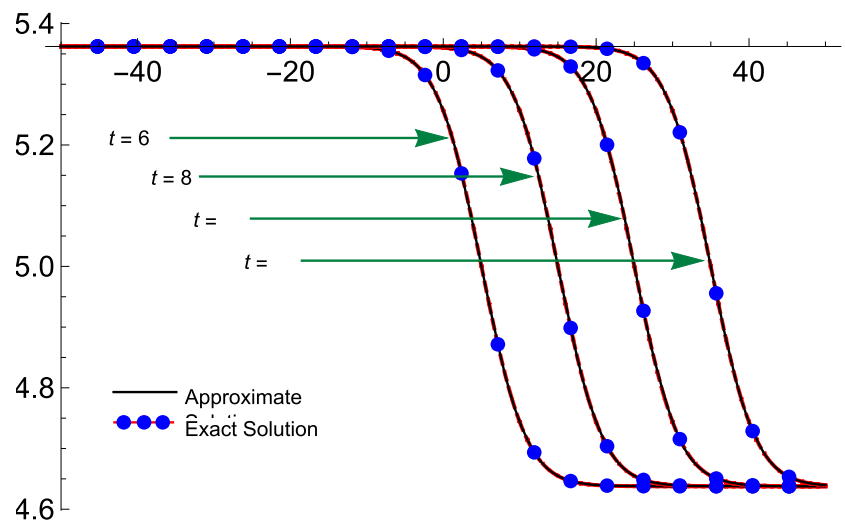

Figure 3 Numerical and analytical exact solutions for Problem 2 with $\Delta t=0.01, n=100, \lambda=5$ and $v=-25$

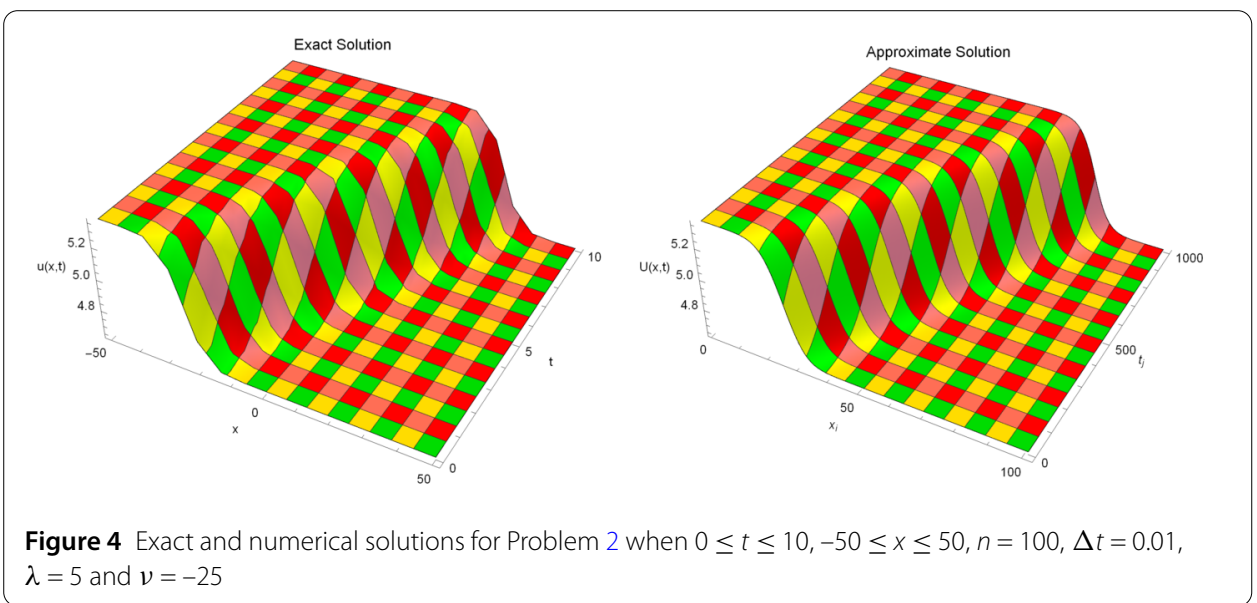

ferent time stages. The 3D graphs of the exact and numerical solutions are shown in Fig. 4.

Problem 3 Consider the following KS equation [29, 33]:

$$
y_{t}+y y_{x}+y_{x x}+0.5 y_{x x x x}=0, \quad x \in[-10,10], t \in[0,10] .
$$


The piecewise defined spline solution at $t=1$ using proposed method for Example 3, when $n=100, \Delta t=0.01$ and $\lambda=0.1$, is given by

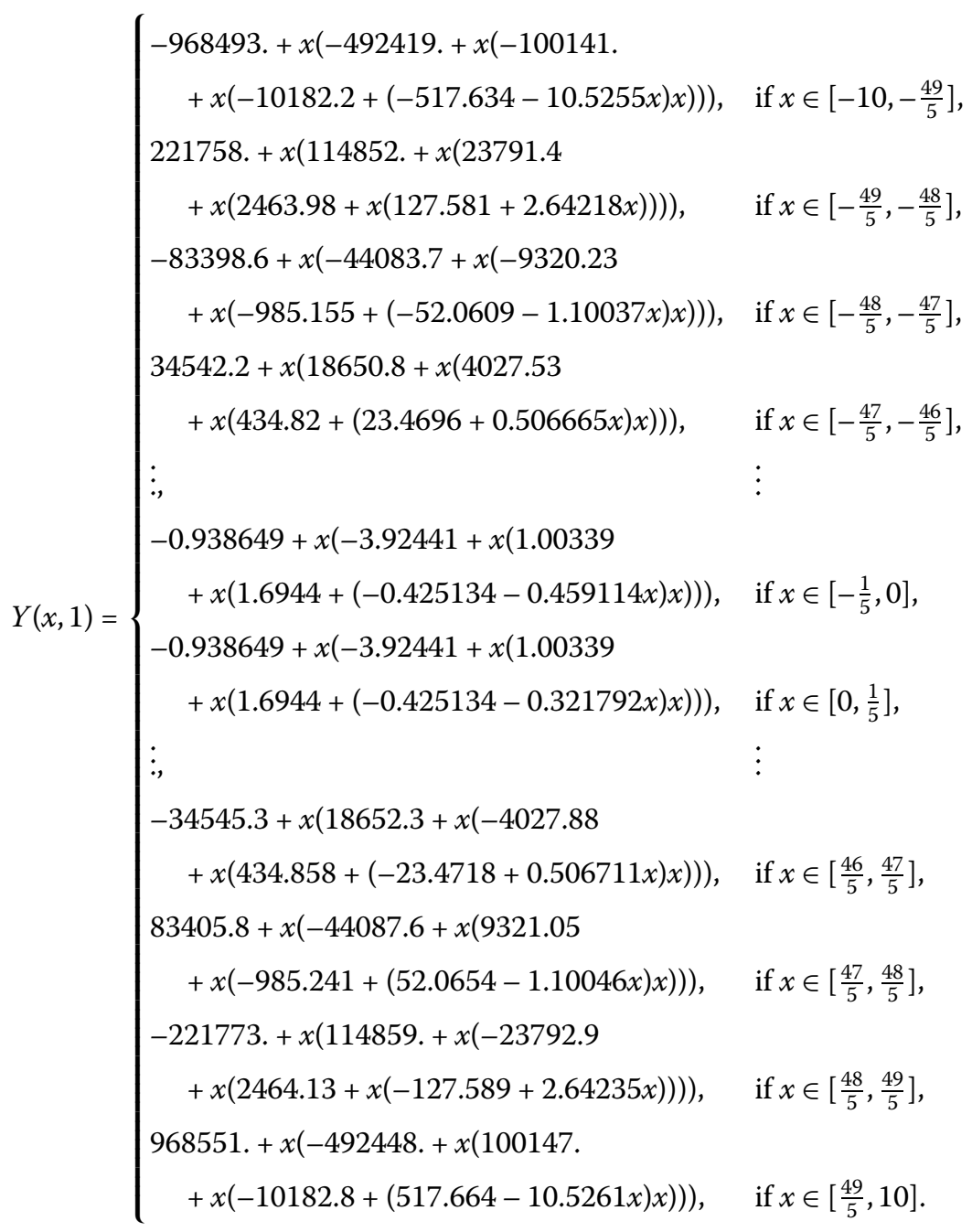

The exact solution is

$$
y(x, t)=-\frac{\lambda}{\mu}+\frac{60}{19} \mu\left(-38 \gamma \mu^{2}+\alpha\right) \tanh (\mu x+\lambda t)+120 \gamma \mu^{3} \tanh ^{3}(\mu x+\lambda t),
$$

where $\mu=\frac{1}{2} \sqrt{11 \alpha / 19 \gamma}$ and the initial and boundary constraints are obtained from the given exact solution. The error norms, $L_{2}$ and $L_{\infty}$, with $\lambda=0.1$ and $\Delta t=0.1,0.01,0.001$ are reported in Table 4. It is clear that our numerical algorithm provides a better approximation to the exact solution than BSF [29] and PSF [33]. The 2D graphs of numerical and true solutions at different time levels are shown in Fig. 5, and Fig. 6 depicts the $3 \mathrm{D}$ plots of the exact and numerical solutions in the temporal domain $0 \leq t \leq 10$ using $\Delta t=0.01$. 
Table 4 Error norms for Problem 3 when $\lambda=0.1$

\begin{tabular}{|c|c|c|c|c|c|c|}
\hline \multirow[t]{2}{*}{$\Delta t$} & \multicolumn{2}{|l|}{ BSF [29] } & \multicolumn{2}{|l|}{ PSF [33] } & \multicolumn{2}{|c|}{ Proposed method } \\
\hline & $\overline{L_{2}}$ & $L_{\infty}$ & $\overline{L_{2}}$ & $L_{\infty}$ & $\overline{L_{2}}$ & $L_{\infty}$ \\
\hline$\overline{0.1}$ & $8.9 \times 10^{-3}$ & $9.9 \times 10^{-6}$ & $1.4 \times 10^{-4}$ & $1.3 \times 10^{-4}$ & $5.83 \times 10^{-5}$ & $7.09 \times 10^{-5}$ \\
\hline 0.01 & $1.6 \times 10^{-3}$ & $2.1 \times 10^{-6}$ & $1.5 \times 10^{-6}$ & $1.3 \times 10^{-6}$ & $5.84 \times 10^{-7}$ & $7.11 \times 10^{-7}$ \\
\hline 0.001 & $\ldots$ & $\ldots$ & $2.2 \times 10^{-8}$ & $2.1 \times 10^{-8}$ & $6.54 \times 10^{-9}$ & $7.95 \times 10^{-9}$ \\
\hline
\end{tabular}

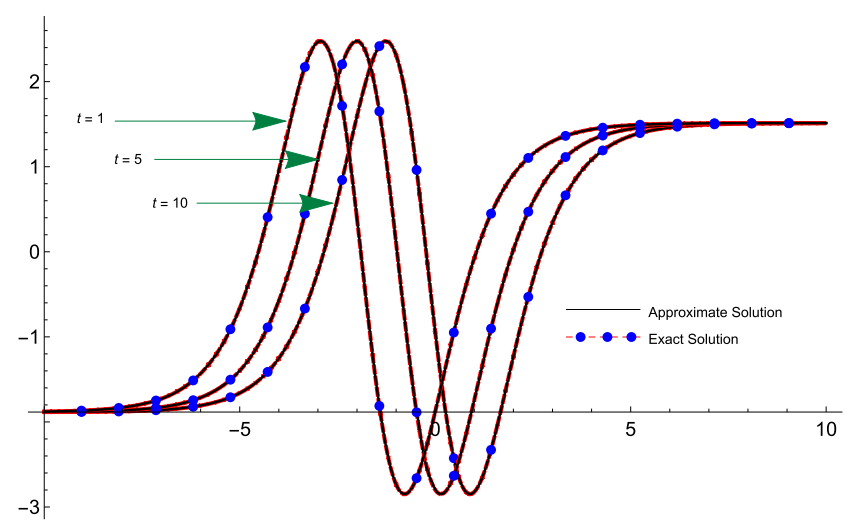

Figure 5 Numerical and exact solutions for Problem 3 at $t=1,5,10$ using $n=100$ and $\Delta t=0.01$

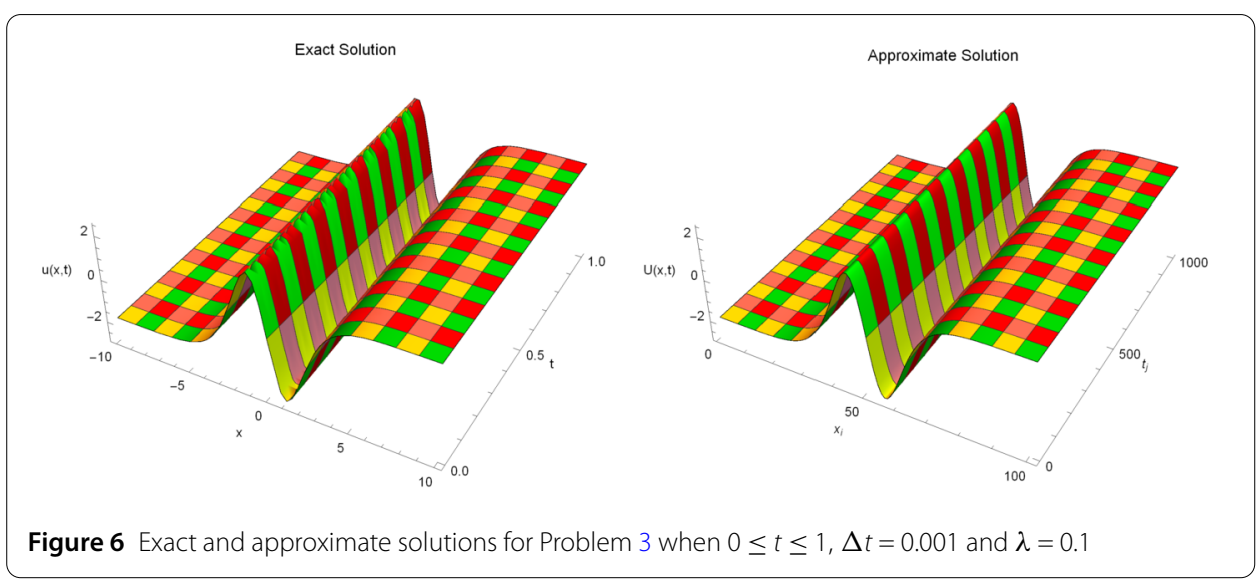

Table 5 Global relative error for Problem 4 when $\lambda=6, \mu=0.5$ and $\nu=-10$

\begin{tabular}{|c|c|c|c|}
\hline$t$ & $\begin{array}{l}\text { LBM [25] } \\
n=600 \\
\Delta t=0.0001\end{array}$ & $\begin{array}{l}\text { QnBS-DQM [6] } \\
n=150\end{array}$ & $\begin{array}{l}\text { Proposed method } \\
n=150 \\
\Delta t=0.001\end{array}$ \\
\hline 1 & $2.59 \times 10^{-2}$ & $2.56 \times 10^{-3}$ & $5.14 \times 10^{-4}$ \\
\hline 2 & $2.80 \times 10^{-2}$ & $4.91 \times 10^{-3}$ & $1.39 \times 10^{-3}$ \\
\hline 3 & $2.67 \times 10^{-2}$ & $1.11 \times 10^{-2}$ & $3.02 \times 10^{-3}$ \\
\hline 4 & $3.52 \times 10^{-2}$ & $1.92 \times 10^{-2}$ & $5.03 \times 10^{-3}$ \\
\hline
\end{tabular}


Problem 4 Consider the KS equation [6, 25]

$y_{t}+y y_{x}+y_{x x}+4 y_{x x x}+y_{x x x x}=0, \quad x \in[-30,30], t \in[0,4]$.

The piecewise defined spline solution at $t=1$ using the proposed method for Example 4, when $n=100, \Delta t=0.01, \lambda=6, \mu=0.5$ and $v=-10$, is given by

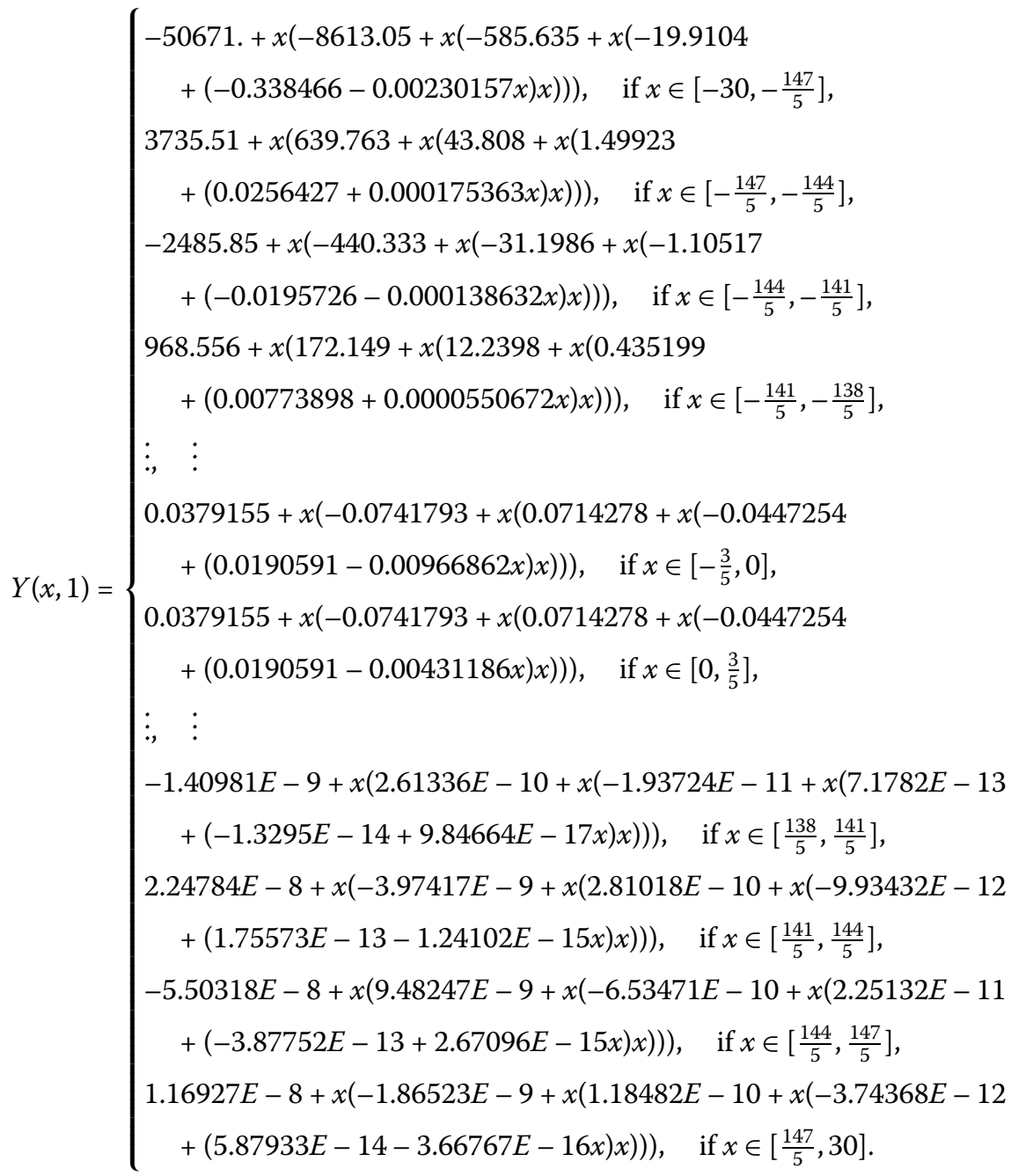

The exact solution is

$$
y(x, t)=9+\lambda-15\left[\operatorname { t a n h } \left(\mu(x-\lambda t-v)+\tanh ^{2}\left(\mu(x-\lambda t-v)+\tanh ^{3}(\mu(x-\lambda t-v)] .\right.\right.\right.
$$

The initial and end conditions are established from given exact solution. Table 5 portrays the comparison of GRE with LBM [25] and QnBS-DQM [6] corresponding to $\lambda=6, \mu=$ $0.5, v=-10, n=150$ and $\Delta t=0.001$ at $t=1,2,3,4$. Figure 7 shows the comparison of approximate and exact solution at different time stages. The 3D graphics of the exact and numerical solutions are given in Fig. 8 when $-30 \leq x \leq 30,0 \leq t \leq 1$ using $n=100$ and $\Delta t=0.01$. 


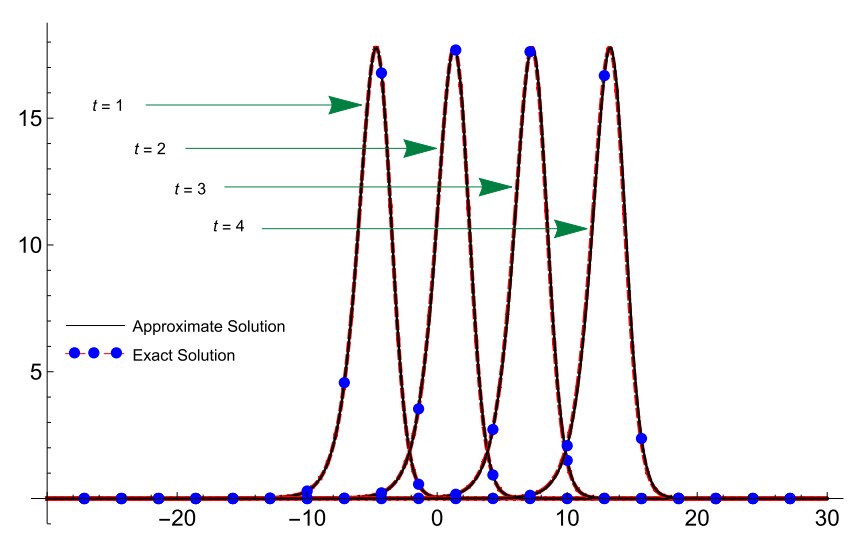

Figure 7 Numerical and analytical exact solutions for Problem 4 with $\Delta t=0.01, n=100, \lambda=6, \mu=0.5$ and $v=-10$

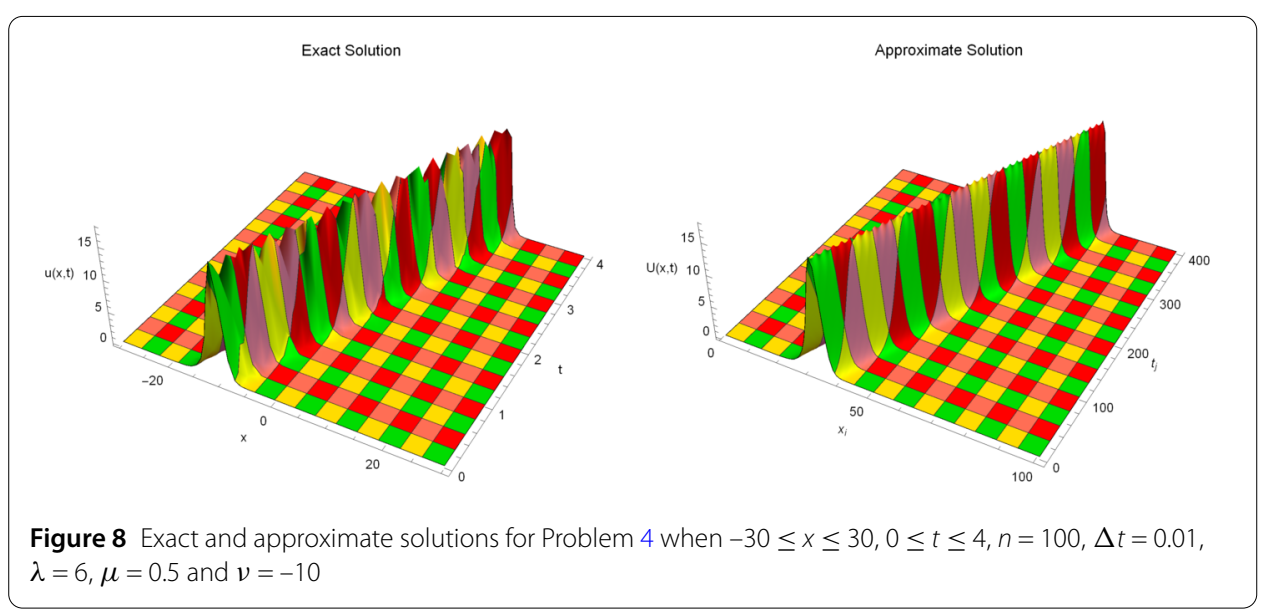

\section{Conclusion}

In this work, an application of a new quintic polynomial B-spline approximation approach has been presented for a numerical investigation of the Kuramoto-Sivashinsky equation. The numerical scheme employs typical fifth degree polynomial basis spline functions in association with a new approximation and a Crank-Nicolson scheme to discretize the problem in the space and time directions, respectively. The error and stability analysis of the proposed scheme is carried out. Four test problems are considered from the available literature and the simulation results are compared with LBM [25], QnBSM [27], BSF [29], ExCBSM [31], QnBS-DQM [6] and PSF [33]. It is concluded that the presented algorithm outperforms the other variants on the topic with superior accuracy and straightforward implementation.

\section{Acknowledgements}

The authors are grateful to the anonymous reviewers for their helpful and valuable comments and suggestions for improvement of this manuscript. We also thank Dr. Muhammad Amin for his assistance in proofreading of the manuscript.

Funding

No funding is available for this research. We are grateful to Springer Open on providing full wavier for this manuscript. 


\section{Competing interests}

The authors declare that they have no competing interests.

\section{Authors' contributions}

All authors equally contributed to this work. All authors read and approved the final manuscript.

\section{Author details}

${ }^{1}$ Department of Mathematics, Government College University, Allama lqbal Road, 38000 Faisalabad, Pakistan.

${ }^{2}$ Department of Mathematics, University of Sargodha, University Road, 40100 Sargodha, Pakistan. ${ }^{3}$ Department of

Software Engineering, Mirpur University of Science \& Technology, College Road, 10250 Mirpur, Azad-Kashmir, Pakistan.

\section{Publisher's Note}

Springer Nature remains neutral with regard to jurisdictional claims in published maps and institutional affiliations.

Received: 6 May 2020 Accepted: 24 September 2020 Published online: 07 October 2020

\section{References}

1. Hooper, A., Grimshaw, R.: Nonlinear instability at the interface between two viscous fluids. Phys. Fluids 28(1), 37-45 (1985)

2. Sivashinsky, G.I.: Instabilities, pattern formation, and turbulence in flames. Annu. Rev. Fluid Mech. 15(1), 179-199 (1983)

3. Conte, R.: Exact solutions of nonlinear partial differential equations by singularity analysis. In: Direct and Inverse Methods in Nonlinear Evolution Equations, Lecture Notes in Physics, vol. 632, pp. 1-83. Springer, Berlin (2003)

4. Hyman, J.M., Nicolaenko, B.: The Kuramoto-Sivashinsky equation: a bridge between PDEs and dynamical systems. Phys. D: Nonlinear Phenom. 18(1-3), 113-126 (1986)

5. Michelson, D.: Steady solutions of the Kuramoto-Sivashinsky equation. Phys. D: Nonlinear Phenom. 19(1), 89-111 (1986)

6. Mittal, R., Dahiya, S.: A quintic B-spline based differential quadrature method for numerical solution of Kuramoto-Sivashinsky equation. Int. J. Nonlinear Sci. Numer. Simul. 18(2), 103-114 (2017)

7. Goufo, E.F.D., Kumar, S., Mugisha, S.: Similarities in a fifth-order evolution equation with and with no singular kernel. Chaos Solitons Fractals 130, 109467 (2020)

8. Ghanbari, B., Kumar, S., Kumar, R.: A study of behaviour for immune and tumor cells in immunogenetic tumour model with non-singular fractional derivative. Chaos Solitons Fractals 133, 109619 (2020)

9. Kumar, S., Kumar, R., Cattani, C., Samet, B.: Chaotic behaviour of fractional predator-prey dynamical system. Chaos Solitons Fractals 135, 109811 (2020)

10. Kumar, S., Kumar, R., Agarwal, R.P., Samet, B.: A study on population dynamics of two interacting species by Haar wavelet and Adam's-Bashforth-Moulton methods. Math. Methods Appl. Sci. 43(8), 5564-5578 (2020)

11. Kumar, S., Ghosh, S., Samet, B., Goufo, E.F.D.: An analysis for heat equations arises in diffusion process using new Yang-Abdel-Aty-Cattani fractional operator. Math. Methods Appl. Sci. 43(9), 6062-6080 (2020)

12. Kumar, S., Ahmadian, A., Kumar, R., Kumar, D., Singh, J., Baleanu, D., Salimi, M.: An efficient numerical method for fractional SIR epidemic model of infectious disease by using Bernstein wavelets. Mathematics 8(4), 558 (2020)

13. Alshabanat, A., Jleli, M., Kumar, S., Samet, B.: Generalization of Caputo-Fabrizio fractional derivative and applications to electrical circuits. Front. Phys. 8, 64 (2020)

14. Kumar, S., Ghosh, S., Lotayif, M.S., Samet, B.: A model for describing the velocity of a particle in Brownian motion by robotnov function based fractional operator. Alex. Eng. J. 59(3), 1435-1449 (2020)

15. Baleanu, D., Jeli, M., Kumar, S., Samet, B.: A fractional derivative with two singular kernels and application to a heat conduction problem. Adv. Differ. Equ. 2020, 252 (2020)

16. Akgül, A.: A novel method for a fractional derivative with non-local and non-singular kernel. Chaos Solitons Fractals $114,478-482$ (2018)

17. Akgül, A.: Reproducing kernel Hilbert space method based on reproducing kernel functions for investigating boundary layer flow of a Powell-Eyring non-Newtonian fluid. J. Taibah Univ. Sci. 13(1), 858-863 (2019)

18. Akgül, A., Karatas Akgül, E.: A novel method for solutions of fourth-order fractional boundary value problems. Fractal Fract. 3(2), 33 (2019)

19. Akgül, A., Cordero, A., Torregrosa, J.R.: A fractional Newton method with $2 \alpha$ th-order of convergence and its stability. Appl. Math. Lett. 98, 344-351 (2019)

20. Akgül, E.K.: Solutions of the linear and nonlinear differential equations within the generalized fractional derivatives. Chaos, Interdiscip. J. Nonlinear Sci. 29(2), 023108 (2019)

21. Soleymani, F., Akgül, A., Akgül, E.K.: On an improved computational solution for the 3D HCIR PDE in finance. An. Ştiinţ. Univ. 'Ovidius' Constanța, Ser. Mat. 27(3), 207-230 (2019)

22. Baleanu, D., Fernandez, A., Akgül, A.: On a fractional operator combining proportional and classical differintegrals. Mathematics 8(3), 360 (2020)

23. Akgül, A., Cordero, A., Torregrosa, J.R.: Solutions of fractional gas dynamics equation by a new technique. Math. Methods Appl. Sci. 43(3), 1349-1358 (2020)

24. Khater, A., Temsah, R.: Numerical solutions of the generalized Kuramoto-Sivashinsky equation by Chebyshev spectral collocation methods. Comput. Math. Appl. 56(6), 1465-1472 (2008)

25. Lai, H., Ma, C.: Lattice Boltzmann method for the generalized Kuramoto-Sivashinsky equation. Phys. A, Stat. Mech. Appl. 388(8), 1405-1412 (2009)

26. Uddin, M., Haq, S.: A mesh-free numerical method for solution of the family of Kuramoto-Sivashinsky equations. Appl. Math. Comput. 212(2), 458-469 (2009)

27. Mittal, R.C., Arora, G.: Quintic B-spline collocation method for numerical solution of the Kuramoto-Sivashinsky equation. Commun. Nonlinear Sci. Numer. Simul. 15(10), 2798-2808 (2010) 
28. Porshokouhi, M.G., Ghanbari, B.: Application of He's variational iteration method for solution of the family of Kuramoto-Sivashinsky equations. J. King Saud Univ., Sci. 23(4), 407-411 (2011)

29. Lakestani, M., Dehghan, M.: Numerical solutions of the generalized Kuramoto-Sivashinsky equation using B-spline functions. Appl. Math. Model. 36(2), 605-617 (2012)

30. Rageha, T.M., Ismaila, H.N., Salemb, G.S., El-Salamc, F.: Restrictive approximation algorithm for Kuramoto-Sivashinsky equation. Int. J. Mod. Math. Sci. 13(1), 29-38 (2015)

31. Ersoy, O., Dag, I.: The exponential cubic B-spline collocation method for the Kuramoto-Sivashinsky equation. Filomat 30(3), 853-861 (2016)

32. Gomes, S.N., Papageorgiou, D.T., Pavliotis, G.A.: Stabilizing non-trivial solutions of the generalized Kuramoto-Sivashinsky equation using feedback and optimal control: Lighthill-Thwaites prize. IMA J. Appl. Math. 82(1), 158-194 (2017)

33. Rashidinia, J., Jokar, M.: Polynomial scaling functions for numerical solution of generalized Kuramoto-Sivashinsky equation. Appl. Anal. 96(2), 293-306 (2017)

34. Akgül, A., Bonyah, E.: Reproducing kernel Hilbert space method for the solutions of generalized Kuramoto-Sivashinsky equation. J. Taibah Univ. Sci. 13(1), 661-669 (2019)

35. Boor, C.D.: A Practical Guide to Splines. Springer, New York (1978)

36. Nazir, T., Abbas, M., lqbal, M.K.: A new quintic B-spline approximation for numerical treatment of Boussinesq equation. J. Math. Comput. Sci. 20(1), 30-42 (2020)

37. Fyfe, D.: The use of cubic splines in the solution of two-point boundary value problems. Comput. J. 12(2), 188-192 (1969)

38. Lodhi, R.K., Mishra, H.K.: Solution of a class of fourth order singular singularly perturbed boundary value problems by quintic B-spline method. J. Niger. Math. Soc. 35(1), 257-265 (2016)

39. Xu, X.P., Lang, F.G.: Quintic B-spline method for function reconstruction from integral values of successive subintervals. Numer. Algorithms 66(2), 223-240 (2014)

40. Iqbal, M.K., Iftikhar, M.W., Iqbal, M.S., Abbas, M.: Numerical treatment of fourth-order singular boundary value problems using new quintic B-spline approximation technique. Int. J. Adv. Appl. Sci. 7(6)، 48-56 (2020)

41. Zin, S.M., Abbas, M., Majid, A.A., Ismail, A.I.M.: A new trigonometric spline approach to numerical solution of generalized nonlinear Klein-Gordon equation. PLoS ONE 9(5), 95774 (2014)

42. Iqbal, M.K., Abbas, M., Wasim, I.: New cubic B-spline approximation for solving third order Emden-Flower type equations. Appl. Math. Comput. 331, 319-333 (2018)

\section{Submit your manuscript to a SpringerOpen ${ }^{\circ}$ journal and benefit from:}

- Convenient online submission

- Rigorous peer review

- Open access: articles freely available online

- High visibility within the field

- Retaining the copyright to your article

Submit your next manuscript at $>$ springeropen.com 